\title{
Testing for unit roots in bounded time series*
}

\author{
Giuseppe Cavaliere \\ Fang Xu \\ University of Bologna \\ University of Reading \\ Forthcoming in Journal of Econometrics
}

\begin{abstract}
Many key economic and financial series are bounded either by construction or through policy controls. Conventional unit root tests are potentially unreliable in the presence of bounds, since they tend to over-reject the null hypothesis of a unit root, even asymptotically. So far, very little work has been undertaken to develop unit root tests which can be applied to bounded time series. In this paper we address this gap in the literature by proposing unit root tests which are valid in the presence of bounds. We present new augmented Dickey-Fuller type tests as well as new versions of the modified ' $M$ ' tests developed by $\mathrm{Ng}$ and Perron (2001, Econometrica 69, pp. 1519-1554) and demonstrate how these tests, combined with a simulation-based method to retrieve the relevant critical values, make it possible to control size asymptotically. A Monte Carlo study suggests that the proposed tests perform well in finite samples. Moreover, the tests outperform the Phillips-Perron type tests originally proposed in Cavaliere (2005, Econometric Theory 21, 907-945). An illustrative application to U.S. interest rate data is provided.
\end{abstract}

\section{Introduction}

In his latest contribution Clive Granger (2010) suggests that the analysis of time series which, despite being non-stationary, are bounded, is a topic which deserves further attention. Specifically, he argues that the unsolved issue is how a concept such as I(1) can be extended to bounded processes.

\footnotetext{
${ }^{*}$ We thank Francis X. Diebold, Jean-Marie Dufour, Helmut Herwartz, Helmut Lütkepohl, Morten Ø. Nielsen, Robert Taylor, two anonymous referees and seminar participants at CREATES (Aarhus), the European University Institute, the London School of Economics, Queen's University, University of Cyprus, University of Montreal and CIREQ, University of Pennsylvania, University of Reading and participants at the Conference in Honour of Clive Granger held in Nottingham, 21-22 May 2010, for useful comments. Cavaliere acknowledges financial support from Italian PRIN 2007 grants. Xu acknowledges financial support from Fritz Thyssen Stiftung (Az.10.08.1.088). Parts of this paper were written while $\mathrm{Xu}$ was a research fellow at the Christian-Albrechts-University of Kiel and a Max Weber Fellow at the European University Institute, and while Cavaliere visited the Economics Department at Queen's University, Canada, whose hospitality is gratefully acknowledged, as a John Weatherall Distinguished Fellow.
} 
According to Granger (2010), 'a limited process is one that has bounds either below (at zero, say) or above (full capacity) or both'. Indeed, many important economic and financial series are bounded in this sense. Examples are expenditure and budget shares, unemployment rates, nominal interest rates, target zone exchange rates. Although limited time series cannot be integrated in the usual sense, see the discussion in Granger (2010), in many theoretical and applied studies they are modelled as pure I(1) processes.

Cavaliere (2005) is the only attempt to explain how the concept of I(1) can coexist with the constraints of a bounded process. He shows that in the presence of (one or two) bounds, the well known Phillips-Perron (1988) [PP] unit root test statistics are characterized by a quite different asymptotic behavior. In general, the limiting null distributions depend upon nuisance parameters related to the position of the bounds: the tighter the bounds, the more shifted to the left the distributions of the unit root statistics. As a consequence, unit root tests based on standard asymptotic critical values become oversized. Only when the bounds are sufficiently far away, conventional unit root methods behave according to the standard asymptotic theory. Cavaliere (2005) also proposes a two-stage procedure where the nuisance parameters related to the bounds are first estimated. These estimates are then employed to retrieve bound-robust (asymptotic) critical values which can be applied to the standard PP tests.

Although it allows to obtain asymptotically valid tests, the approach proposed in Cavaliere (2005) suffers from the well-known finite sample size problems affecting PP unit root tests as well as most of the tests based on sum-of-covariances estimators of the long-run variance. More robust approaches, such as tests based on spectral estimators of the long run variance ( $\mathrm{Ng}$ and Perron, 1995, 2001) or the well-known Said-Dickey-Fuller [ADF] tests, could be applied. Unfortunately, no theory for such tests is available for bounded time series.

In this paper we aim at filling this gap by proposing a new approach to unit root testing in bounded time series, which leads to tests being asymptotically valid and possessing good finite sample properties. By focusing on the ADF tests as well as on the autocorrelation-robust ' $M$ ' unit root tests of Perron and Ng (1996), Stock (1999) and Ng and Perron (2001) - although the approach we outline can equally well be applied to any of the commonly used unit root statistics - we propose a numerical solution to the inference problem. Specifically, direct simulation methods - based on new consistent estimators of the nuisance parameters related to the bounds - are employed to obtain approximate $p$-values from the asymptotic null distributions of the standard unit root statistics. A variety of algorithms are also suggested to account for potential 
autocorrelation and heteroskedasticity in the error terms. In addition, we demonstrate that the simulation-based $A D F$ and $M$ tests possess good finite sample properties, outperforming the PP tests considered in Cavaliere (2005).

As for the test discussed in Cavaliere (2005), our tests can be applied to series which have either one bound (above, or below, such as for the much discussed case of nominal interest rates) or two bounds. Moreover, we allow the errors to be general linear processes driven by martingale difference innovations, hence allowing for conditional heteroskedasticity e.g. of the ARCH type.

The paper is organized as follows. The next section introduces bounded integrated processes and discusses the main assumptions. In Section 3 the asymptotic distributions of the $A D F$ and $M$ test statistics are derived and their dependence on nuisance parameters related to the position of the bounds is established. The simulation-based unit root tests that account for the presence of bounds are presented in Section 4. The finite sample properties are investigated in Section 5. A brief illustrative application to US interest rates is reported in Section 6. Section 7 concludes. All proofs are collected in the Appendix. The following notation is used through out the paper. ' $\lfloor\cdot\rfloor$ ' denotes the integer part of its argument; ' $\stackrel{w}{\rightarrow}$ ' denotes weak convergence and ' $\stackrel{p}{\rightarrow}$ ' convergence in probability, in each case as the sample size diverges to positive infinity; ' $x:=y$ ' (' $\left.x=: y^{\prime}\right)$ indicates that $x$ is defined by $y$ ( $y$ is defined by $x) ; \mathcal{D}:=D[0,1]$ is the space of right continuous with left limit (càdlàg) processes on $[0,1]$, equipped with the Skorohod metric; $\|x\|$ denotes the standard Euclidean norm of a column vector $x$; the norm of a matrix $B$ is defined as $\|B\|=\sup _{\|x\|<1}\|B x\|$, and $\mathbb{I}(\cdot)$ is the indicator function.

\section{Bounded unit root processes}

This section introduces the reference class of bounded non-stationary processes. We consider processes that behave similarly to random walks but, at the same time, are bounded either above or below, or both. Processes belonging to this class will be referred to as 'bounded I(1)' or 'bounded unit root' processes, BI(1) hereafter. Bounded I(1) processes are discussed in Cavaliere (2005) and Granger (2010).

A bounded time series $X_{t}$, with (fixed) bounds at $\underline{b}, \bar{b}(\underline{b}<\bar{b})$, is a stochastic process satisfying $X_{t} \in[\underline{b}, \bar{b}]$ almost surely for all $t$. This requires that, at each $t$, the increment $\Delta X_{t}$ lies within the interval $\left[\underline{b}-X_{t-1}, \bar{b}-X_{t-1}\right]$. Focusing on the case of a constant deterministic component, a simple and relatively general way to extend the notion of bounded processes to the unit root case 
is to assume that (see Cavaliere, 2005)

$$
\begin{aligned}
X_{t} & =\theta+Y_{t} \\
Y_{t} & =\alpha Y_{t-1}+u_{t}, \alpha=1
\end{aligned}
$$

initialized at $Y_{0}=O_{p}(1)$. The term $u_{t}$ is further decomposed as follows:

$$
u_{t}=\varepsilon_{t}+\underline{\xi}_{t}-\bar{\xi}_{t}
$$

where $\varepsilon_{t}$ is a (weakly dependent) zero-mean unbounded process and $\underline{\xi}_{t}, \bar{\xi}_{t}$ are non-negative processes such that $\underline{\xi}_{t}>0$ if and only if $Y_{t-1}+\varepsilon_{t}<\underline{b}-\theta$ and, similarly, $\bar{\xi}_{t}>0$ if and only if $Y_{t-1}+\varepsilon_{t}>\bar{b}-\theta$. Since any truncated, censored or reflected random variable can be represented as in (2.3) for some unbounded $\varepsilon_{t}$ (see the discussion in Cavaliere, 2005), this assumption is quite general.

A $\mathrm{BI}(1)$ process reverts because of the bounds only. It behaves as a unit root process when it is far away from the bounds. Conversely, in the neighborhood of the bounds it differs from standard I(1) processes because of the presence of the terms $\underline{\xi}_{t}$ and $\bar{\xi}_{t}$, which force $X_{t}$ to lie between $\underline{b}$ and $\bar{b}$. In the stochastic control literature, see Harrison (1985), $\underline{\xi}_{t}$ and $\bar{\xi}_{t}$ are referred to as 'regulators', as they control the path of $X_{t}$ by keeping it between $\underline{b}$ and $\bar{b}$.

Throughout the paper we assume that $\varepsilon_{t}$ is a general linear process [LP] of the form

$$
\varepsilon_{t}=C(L) v_{t}
$$

where $v_{t}$ is a martingale difference sequence $[\mathrm{MDS}]$ and $C(z):=\sum_{j=0}^{\infty} c_{j} z^{j}$. We make use of the following standard assumption on $\varepsilon_{t}$, see e.g. Chang and Park (2002, pp.433-4).

Assumption $\mathcal{A}: \mathcal{A}_{1}$. (a) $\left\{v_{t}, \mathcal{F}_{t}\right\}$ is a MDS with respect to some filtration $\mathcal{F}_{t}$, such that $E\left(v_{t}^{2}\right)=$ $\sigma^{2}<\infty$, (b) $T^{-1} \sum_{t=1}^{T} v_{t}^{2} \stackrel{p}{\rightarrow} \sigma^{2}$, and (c) $E\left|v_{t}\right|^{r}<\infty$ for some $r \geq 4 ; \mathcal{A}_{2}$. The lag polynomial satisfies $C(z) \neq 0$ for all $|z| \leq 1$ and $\sum_{j=0}^{\infty} j^{s}\left|c_{j}\right|<\infty$ for some $s \geq 1$.

In addition, we consider two further conditions related to the bounds. The first is a technical condition needed to prevent $\left\{X_{t}\right\}$ from 'jumping' too much at the bounds. The second condition allows to treat the positions of the upper and of the lower bounds as nuisance parameters. Moreover, under $\mathcal{B}_{2}$ the presence of the bounds does not annihilate the $\sim T^{1 / 2}$ order of magnitude of the random walk component of the data (see below). 
Assumption $\mathcal{B}: \mathcal{B}_{1} \cdot \sup _{t=1, \ldots, T} E\left|\underline{\xi}_{t}\right|^{r}<\infty$ and $\sup _{t=1, \ldots, T} E\left|\bar{\xi}_{t}\right|^{r}<\infty$, with $r$ given in $\mathcal{A}_{1}$; $\mathcal{B}_{2} .(\underline{b}-\theta) /\left(\lambda T^{1 / 2}\right)=\underline{c}+o(1)$ and $(\bar{b}-\theta) /\left(\lambda T^{1 / 2}\right)=\bar{c}+o(1)$, where $\underline{c} \leq 0 \leq \bar{c}, \underline{c} \neq \bar{c}$, and $\lambda^{2}:=\sigma^{2} C(1)^{2}$ denotes the long-run variance of $\varepsilon_{t}$.

Some remarks are due.

Remark 2.1. Under Assumption $\mathcal{A}, C(z)^{-1}=: \alpha(z)=1-\sum_{j=1}^{\infty} \alpha_{j} z^{j}$ is well defined. By letting $\underline{\xi}_{t}^{*}:=\alpha(L) \underline{\xi}_{t}$ and $\bar{\xi}_{t}^{*}:=\alpha(L) \bar{\xi}_{t}$ we can write

$$
u_{t}=C(L) v_{t}+\underline{\xi}_{t}-\bar{\xi}_{t}=C(L) v_{t}^{*}, v_{t}^{*}:=v_{t}+\underline{\xi}_{t}^{*}-\bar{\xi}_{t}^{*}
$$

The differenced process $\Delta X_{t}$ therefore admits the LP representation $\Delta X_{t}=C(L) v_{t}^{*}$. Different from the standard $\mathrm{I}(1)$ case, $v_{t}^{*}$ depends both on the innovations $\varepsilon_{t}$ and the (current and past) regulators, $\underline{\xi}_{t}$ and $\bar{\xi}_{t}$.

Remark 2.2. As is standard, via the Beveridge-Nelson [BN] representation (cf. Phillips and Solo, 1992) $\varepsilon_{t}$ can be written as $\varepsilon_{t}=C(1) v_{t}+\tilde{\varepsilon}_{t-1}-\tilde{\varepsilon}_{t}$, with $\tilde{\varepsilon}_{t}=\sum_{j=0}^{\infty} \tilde{c}_{j} v_{t-j}\left(\tilde{c}_{j}:=\sum_{i=j+1}^{\infty} c_{i}\right)$ being well defined in the $L^{r}$ sense. Consequently, $X_{t}$ can be decomposed as

$$
X_{t}=\theta+C(1) \sum_{i=1}^{t} v_{i}+\sum_{i=1}^{t}\left(\underline{\xi}_{i}-\bar{\xi}_{i}\right)+\tilde{\varepsilon}_{0}-\tilde{\varepsilon}_{t} .
$$

Eq. (2.6) implies that the non-stationary component of the $\mathrm{BI}(1)$ process can be decomposed into a standard random walk, $\sum_{i=1}^{t} v_{i}$, and the cumulated regulators, $\sum_{i=1}^{t}\left(\underline{\xi}_{i}-\bar{\xi}_{i}\right)$. Under Assumption $\mathcal{B}_{2}$, these two terms are of the same order $\left(\sim T^{1 / 2}\right)$. Therefore, the behavior of $X_{t}$ is influenced by the regulators not only at short horizons, but in the long run as well. Notice also that, due to the presence of the cumulated regulators, standard convergence tools for I(1) processes (such as FCLTs) are not expected to hold in general.

REMARK 2.3. For the unit root tests which will be discussed later it is useful to notice that an autoregressive $[\mathrm{AR}]$ approximation can be given to $u_{t}$. Since $(2.5)$ implies the $\operatorname{AR}(\infty)$ representation $\alpha(L) u_{t}=v_{t}^{*}$, we may write

$$
u_{t}=\sum_{i=1}^{k} \alpha_{i} u_{t-i}+v_{t, k}, v_{t, k}:=v_{t}^{*}+\sum_{i=k+1}^{\infty} \alpha_{i} u_{t-i}
$$

As in Chang and Park (2002, p.434), the moment restrictions on $v_{t}, \bar{\xi}_{t}, \underline{\xi}_{t}$ and the decaying rate for the coefficients of $C(z)$ (cf. Assumptions $\mathcal{A}$ and $\mathcal{B}$ ) imply that the approximation error, i.e. $v_{t, k}-v_{t}^{*}$, satisfies $E\left|v_{t, k}-v_{t}^{*}\right|^{r}=o\left(k^{-r s}\right)$, even in the presence of the regulators $\underline{\xi}_{t}, \bar{\xi}_{t}$. 
REMARK 2.4. Assumption $\mathcal{B}_{2}$ relates the position of the bounds $\underline{b}$ and $\bar{b}$ (relatively to the location parameter $\theta$ ) to the sample size T. As noted in Granger (2010, p.4), 'the qualifying feature of an I(1) process is the strong relationship between now and the distant past, so that $\operatorname{corr}\left(X_{t}, X_{t-k}\right)=1$ for any $k^{\prime}$. Under Assumption $\mathcal{B}_{2}$, this fundamental property is not altered by the presence of the bounds. Additionally, it is a key condition: (i) for establishing the asymptotic behavior of any unit root test statistics in the presence of bounds, see Cavaliere (2005), and (ii) for constructing proper unit root tests that take account of the bounds without making any parametric assumption on the behavior of $X_{t}$ near the bounds.

REMARK 2.5. One-sided bounds can be treated as a special case by setting $\bar{c}=\infty$ (lower bound only) or $\underline{c}=-\infty$ (upper bound only). By construction, $\bar{c}=\infty(\underline{c}=-\infty)$ implies that the upper regulator $\bar{\xi}_{t}$ (the lower regulator $\underline{\xi}_{t}$ ) equals zero, almost surely, for all $t$.

Remark 2.6. Since under $\mathcal{B}_{2}$ the bound parameters $\underline{b}$ and $\bar{b}$ depends on $T, X_{t}$ formally constitutes a triangular array of the type $\left\{X_{T t}: t=0,1, \ldots, T ; T=0,1, \ldots\right\}$. The double index notation is not essential and bounded integrated processes will be simply denoted as $\left\{X_{t}\right\}$.

\section{Unit root asymptotics for bounded processes}

In this section we discuss how the presence of bounds affects the asymptotic null distribution of the well-known augmented (Said-)Dickey-Fuller tests and of the $M$ unit root tests.

For a given sample $\left\{X_{t}\right\}_{0}^{T}$, the ADF statistics are based on the OLS regression

$$
\hat{X}_{t}=\alpha \hat{X}_{t-1}+\sum_{i=1}^{k} \alpha_{i} \Delta \hat{X}_{t-i}+\varepsilon_{t, k}
$$

and are defined as

$$
\mathcal{A D F}_{\alpha}:=\frac{T(\hat{\alpha}-1)}{\hat{\alpha}(1)}, \mathcal{A D F}_{t}:=\frac{\hat{\alpha}-1}{s(\hat{\alpha})}
$$

where $\hat{\alpha}(1):=1-\sum_{i=1}^{k} \hat{\alpha}_{i}$, with $\hat{\alpha}_{i}$ denoting the OLS estimator of $\alpha_{i}$ in (3.7) and $s(\hat{\alpha})$ the usual OLS standard error of $\hat{\alpha}$. Here $\hat{X}_{t}$ denotes the OLS residuals from the regression of $X_{t}$ on a constant term. Alternatively $\hat{X}_{t}$ can be taken as the pseudo GLS de-meaned series, see Elliott, Rothenberg and Stock (1996).

The $M$ statistics are defined as

$$
\mathcal{M Z}_{\alpha}:=\frac{T^{-1} \hat{X}_{T}^{2}-T^{-1} \hat{X}_{0}^{2}-s_{A R}^{2}(k)}{2 T^{-2} \sum_{t=1}^{T} \hat{X}_{t-1}^{2}}, \mathcal{M S B}:=\left(T^{-2} \sum_{t=1}^{T} \hat{X}_{t-1}^{2} / s_{A R}^{2}(k)\right)^{1 / 2}
$$


and $\mathcal{M Z}_{t}:=\mathcal{M Z}_{\alpha} \times \mathcal{M S B},{ }^{1}$ where $s_{A R}^{2}(k)$ is an autoregressive estimator of the (non-normalized) spectral density at frequency zero of $\left\{u_{t}\right\}$. Specifically,

$$
s_{A R}^{2}(k):=\hat{\sigma}^{2} / \hat{\alpha}(1)^{2}
$$

where $\hat{\alpha}(1)$ is as defined above and $\hat{\sigma}^{2}$ is the OLS variance estimator from the ADF regression (3.7). For all tests the lag truncation parameter is required to satisfy the following assumption (Lewis and Reinsel, 1985).

Assumption $\mathcal{K}$. As $T \rightarrow \infty, 1 / k+k^{2} / T \rightarrow 0$.

It is well known that if Assumptions $\mathcal{A}$ and $\mathcal{B}$ hold on (2.1)-(2.2) and if $X_{t}$ is unbounded (i.e., $\bar{b}=-\underline{b}=\infty$ ), the asymptotic (null) distributions of the $\mathcal{A D F}$ and $M$ statistics are as follows (see Ng and Perron, 2001; Chang and Park, 2002):

$$
\begin{aligned}
\mathcal{A D F}_{\alpha}, \mathcal{M Z}_{\alpha} & \stackrel{w}{\rightarrow} \frac{1}{2}\left(F_{B}(1)^{2}-F_{B}(0)^{2}-1\right)\left(\int_{0}^{1} F_{B}(s)^{2} d s\right)^{-1}=: \zeta_{1} \\
\mathcal{M S B} & \stackrel{w}{\rightarrow}\left(\int_{0}^{1} F_{B}(s)^{2} d s\right)^{1 / 2}=: \zeta_{2} \\
\mathcal{A D F}_{t}, \mathcal{M Z}_{t} & \stackrel{w}{\rightarrow} \zeta_{3}=: \zeta_{1} \zeta_{2}
\end{aligned}
$$

with $F_{B}:=B-\int_{0}^{1} B(r) d r, B$ being a standard Brownian motion. In the case of the $\mathcal{A D F}$ and $\mathcal{M Z}$ tests, the unit root null is rejected for large negative values of the statistics, while a test based on $\mathcal{M S B}$ rejects for small values of the statistic.

In Theorem 1 below we now provide representations for the asymptotic null distributions of the test statistics considered in the presence of bounds. A key role in the asymptotic distributions of the statistics is played by the process $B_{\underline{c}}^{\bar{c}}$, a Brownian motion, regulated at $\underline{c}, \bar{c}$. The regulated Brownian motion $[\mathrm{RBM}]$ behaves like a standard BM except in the neighborhood of the bounds, where it is forced to revert; see Harrison (1985) or Cavaliere (2005) for a technical definition.

Theorem 1 Let $\left\{X_{t}\right\}_{0}^{T}$ be generated as in (2.1) with $\alpha=1$, under Assumptions $\mathcal{A}$ and $\mathcal{B}$. Then: (i) $T^{-1 / 2} Y_{\lfloor T \cdot\rfloor}:=T^{-1 / 2} \sum_{t=1}^{\lfloor T \cdot\rfloor} u_{t} \stackrel{w}{\rightarrow} \lambda B_{\underline{c}}^{\bar{c}}(\cdot)$ in $\mathcal{D}$; (ii) if Assumption $\mathcal{K}$ also holds, $s_{A R}^{2}(k) \stackrel{p}{\rightarrow}$ $\lambda^{2}:=\sigma^{2} C(1)^{2}, \mathcal{A D F}_{\alpha}, \mathcal{M Z}_{\alpha} \stackrel{w}{\rightarrow} 0.5\left(F_{B_{\underline{c}}^{\bar{c}}}(1)^{2}-F_{B_{\underline{c}}^{\bar{c}}}(0)^{2}-1\right)\left(\int_{0}^{1} F_{B_{\underline{c}}^{\bar{c}}}(s)^{2} d s\right)^{-1}=: \zeta_{1}^{\underline{c}, \bar{c}}, \mathcal{M S B} \stackrel{w}{\rightarrow}$ $\left(\int_{0}^{1} F_{B_{\underline{c}}^{\bar{c}}}(s)^{2} d s\right)^{1 / 2}=: \zeta_{\bar{c}}^{\underline{c}, \bar{c}}$, and $\mathcal{A} \mathcal{D} \mathcal{F}_{t}, \mathcal{M Z}_{t} \stackrel{w}{\rightarrow} \zeta_{\bar{c}}^{\underline{c}, \bar{c}}=: \zeta_{\overline{1}}^{\underline{c}, \bar{c}} \zeta_{\overline{2}}^{\underline{c}, \bar{c}}$, where $F_{B_{\underline{c}}^{\bar{c}}}:=B_{\underline{c}}^{\bar{c}}-\int_{0}^{1} B_{\underline{c}}^{\bar{c}}(s) d s$.

The following remarks collect some of the implications of Theorem 1.

\footnotetext{
${ }^{1}$ As in Müller and Elliott (2003), we include the term $-T^{-1} \hat{X}_{0}^{2}$ in the numerator of $\mathcal{M} \mathcal{Z}_{\alpha}$ and $\mathcal{M Z}_{t}$, so that the $\mathcal{A D F}_{\alpha}\left(\mathcal{A D F}_{t}\right)$ and the $\mathcal{M Z}_{\alpha}\left(\mathcal{M Z}_{t}\right)$ statistics have the same limiting distributions.
} 
REMARK 3.1. The results in Theorem 1 differ from standard I(1) asymptotics mainly because the limiting process is not a standard Brownian motion, but a regulated Brownian motion. The sample paths of the limiting process are therefore bounded between $\underline{c}$ and $\bar{c}$, with the well known case of no bounds following as a special case by setting $-\underline{c}$ and $\bar{c}$ equal to infinity.

REMARK 3.2. Under Assumption $\mathcal{B}$, the usual spectral estimator of the long run variance is still consistent for $\lambda^{2}$, the long-run variance of $\varepsilon_{t}$. However, this result alone does not guarantee that the unit root statistics have the usual Dickey-Fuller type distributions. Specifically, the asymptotic distributions $\zeta_{i}^{c}, \bar{c}, i=1,2,3$, are non-standard and depend on the nuisance parameters $\underline{c}, \bar{c}$. Therefore, inference based on the usual quantiles is generally invalid. Only for bounds sufficiently far away the quantiles of the distributions in Theorem 1 are well approximated by the quantiles of $\zeta_{i}, i=1,2,3$. Conversely, unit root tests based on standard critical values are oversized, with the degree of oversizing depending on the two parameters $\underline{c}, \bar{c}$ (the narrower the limits, the higher the degree of oversizing).

REMARK 3.4. When pseudo-GLS de-meaning is used and $X_{t}$ is unbounded, the results in (3.9) hold with $F_{B}$ replaced by $B$. Similarly, in the bounded case Theorem 1 can be generalized to tests based on pseudo-GLS de-trending. In the case of GLS de-meaned data, results for the $A D F$ and $M$ tests are as those given in Theorem 1 but with $B_{\underline{c}}^{\bar{c}}$ replacing $F_{B_{\underline{c}}^{\bar{c}}}$.

REMARK 3.5. The results given in Theorem 1 can be readily extended to the near-integrated case, $\alpha:=1-\kappa / T, 0<\kappa<\infty$ in (2.1)-(2.2). It is straightforward to demonstrate that Theorem 1 continues to hold but with $B_{\underline{c}}^{\bar{c}}$ replaced by the Ornstein-Uhlenbeck [OU] process, $J^{\kappa}(s):=$ $\int_{0}^{s} \exp (-\kappa(s-r)) d B(r)$, regulated at $\underline{c}, \bar{c}$ (see also Cavaliere, 2005, Theorem 4). Consequently, the asymptotic local power function of the unit root tests will also be affected by the bounds.

\section{Testing for unit roots in the presence of bounds}

As discussed in the previous section, standard unit root inference is affected by the presence of bounds since the null asymptotic distributions of the commonly employed test statistics are nonstandard and lead to oversized tests. Hence, in the presence of bounds, where the null hypothesis is rejected on the basis of standard critical values, it is not possible to assess whether the rejection depends on the absence of a unit root or, conversely, on the presence of the bounds only.

Despite the fact that the asymptotic distributions of the unit root test statistics depend on the two nuisance parameters $\underline{c}$ and $\bar{c}$, see Theorem 1 , in this section we are able to propose a simulation-based approach which allows to obtain proper asymptotic $p$-values for unit root tests 
when the time series of interest is bounded. Both one-sided and two-sided bounds are covered.

The derivation of unit root tests for bounded time series takes two steps. First (Section 4.1), we construct two simple, consistent estimators of the nuisance parameters $\underline{c}$ and $\bar{c}$. Second (Sections 4.2 and 4.3), we define a simulation-based approach which draws on such estimators and can be used to retrieve the relevant $p$-values. Extensions to cases of (unconditionally) heteroskedastic shocks are discussed in Section 4.4.

\subsection{Consistent estimation of the bound parameters}

Since the bounds $\underline{b}, \bar{b}$ are assumed to be known, consistent estimation of the nuisance parameters $\underline{c}, \bar{c}$ is actually feasible. To this aim, it suffices to define the following estimators

$$
\widehat{\widehat{c}}:=\frac{\underline{b}-X_{0}}{s_{A R}(k) T^{1 / 2}}, \widehat{\bar{c}}:=\frac{\bar{b}-X_{0}}{s_{A R}(k) T^{1 / 2}}
$$

where $s_{A R}^{2}(k)$ is the spectral AR estimator of the long run variance as defined in Section 2. The main result on the consistency of $\underline{\widehat{c}}$ and $\widehat{\bar{c}}$ is given in the next lemma, which generalizes Corollary 5 of Cavaliere (2005) to the present framework.

Lemma 1 Let the assumptions of Theorem 1 hold. Then, $\underline{\widehat{c}} \stackrel{p}{\rightarrow} \underline{c}, \widehat{\bar{c}} \stackrel{p}{\rightarrow} \bar{c}$.

Hence, given that the bounds $(\underline{b}, \bar{b})$ are known, the two nuisance parameters of the limiting distributions in Theorem 1, $\underline{c}$ and $\bar{c}$, can be consistently estimated via $\underline{\widehat{c}}$ and $\widehat{\bar{c}}$, respectively. These estimators are the two key ingredients for our simulation-based tests.

REMARK 4.1. Notice that in (4.10) the deterministic term $\theta$ is implicitly estimated under the null, as advocated in e.g. Schmidt and Phillips (1992). If $\theta$ were estimated by standard OLS, i.e. by replacing $X_{0}$ of (4.10) by $T^{-1} \sum_{t=1}^{T} X_{t}$, the resulting estimators of $\underline{c}, \bar{c}$ would become inconsistent.

\subsection{Simulation-based tests}

In this section we show how direct simulation methods can be used to retrieve $p$-values from the limiting null distributions of the standard $A D F$ and $M$ statistics given in Theorem 1.

As noted in Section 3, the limiting distributions depend on the regulated Brownian motion,

$B_{\underline{c}}^{\bar{c}}$. Our method is based on the construction of a càdlàg process $B_{n}^{*}$ that satisfies $B_{n}^{*} \stackrel{w}{\rightarrow} B_{\underline{c}}^{\bar{c}}$ with probability tending to one. We can then approximate quantiles from the non-pivotal limiting null 
distributions in Theorem 1 by simple numerical simulation methods based on approximating the limiting process $B_{c}^{\bar{c}}$ through the càdlàg process $B_{n}^{*}$. The simulation-based versions of the $A D F$ and $M$ tests, which we denote generically as $A D F^{*}$ and $M^{*}$ in what follows, only require the computation of the standard $A D F$ and $M$ statistics of Section 3 and of the associated Monte Carlo $[\mathrm{MC}] p$-values. Taking the $A D F_{\alpha}$ test to illustrate, the simulation-based test is constructed according to the following algorithm.

Algorithm 1 Step (i). Let $\varepsilon_{t}^{*}$ be an i.i.d. $(0,1)$ sequence (independent of $\left(X_{0}, \ldots, X_{T}\right)$ );

Step (ii). For some $n \geq T$, let $X_{t}^{*}, t=1, \ldots, n$ be recursively defined as

$$
X_{t}^{*}:=\left\{\begin{array}{cc}
\widehat{\bar{c}} & \text { if } X_{t-1}^{*}+n^{-1 / 2} \varepsilon_{t}^{*}>\widehat{\bar{c}} \\
\widehat{\widehat{c}} & \text { if } X_{t-1}^{*}+n^{-1 / 2} \varepsilon_{t}^{*}<\widehat{\widehat{c}} \\
X_{t-1}^{*}+n^{-1 / 2} \varepsilon_{t}^{*} & \text { otherwise }
\end{array}\right.
$$

with initial condition $X_{0}=0$. The corresponding càdlàg process is $X_{n}^{*}(s):=X_{\lfloor n s\rfloor}^{*}, s \in[0,1]$.

Step (iii). Compute the MC statistics

$$
\mathcal{A D F}_{\alpha}^{*}:=\frac{\tilde{X}_{n}^{*}(1)^{2}-\tilde{X}_{n}^{*}(0)^{2}-1}{2 \int_{0}^{1} \tilde{X}_{n}^{*}(s)^{2} d s}, \tilde{X}_{n}^{*}(s):=X_{n}^{*}(s)-\int_{0}^{1} X_{n}^{*}(u) d u
$$

Step (iv). Define the $M C$ p-value as $p_{n}^{*}:=G_{n}^{*}\left(\mathcal{A D} \mathcal{F}_{\alpha}\right)$, where $G_{n}^{*}$ denotes the cumulative distribution function of $\mathcal{A D} \mathcal{F}_{\alpha}^{*}$, conditional on $\underline{\widehat{c}}, \widehat{\bar{c}}$. Similarly, for any significance level $\eta$, $c v_{\eta}$ that solves $G_{n}^{*}\left(c v_{\eta}\right)=\eta$ is the Monte Carlo critical value.

The following theorem holds as $T$ diverges.

Theorem 2 Let $\left\{X_{t}\right\}_{0}^{T}$ be generated as in (2.1) with $\alpha=1$, under Assumptions $\mathcal{A}, \mathcal{B}$ and $\mathcal{K}$. Then, as $T \rightarrow \infty$ : (i) $X_{n}^{*} \stackrel{w}{\rightarrow} B_{\underline{c}}^{\bar{c}}$ in probability, and (ii) $\mathcal{A D} \mathcal{F}_{\alpha}^{*} \stackrel{w}{\rightarrow} \zeta_{\stackrel{c}{1}, \bar{c}}^{\text {in }}$ probability. Finally, (iii) $p_{n}^{*} \stackrel{w}{\rightarrow} U[0,1]$.

Theorem 2(i)-(ii) shows that for $T$ diverging to infinity, the simulated process $X_{n}^{*}$ is distributed as the limiting process $B_{\underline{c}}^{\bar{c}}$ of Theorem 1(i) and that the MC statistic $\mathcal{A D F}_{\alpha}^{*}$ is asymptotically distributed as $\mathcal{A D F} \mathcal{F}_{\alpha}$ under the unit root null hypothesis. Consequently, see (iii), even if the unit root statistics are not pivotal in the presence of bounds, the $\mathcal{A D F}_{\alpha}^{*}$ test has correct (asymptotic) size. That is, for any chosen significance level $\eta$, as $T$ diverges it holds that $P\left(p_{n}^{*} \leq \eta\right) \rightarrow \eta$ and a test which rejects the null hypothesis when $p_{n}^{*} \leq \eta$ has asymptotic size equal to $\eta$. Some remarks are due. 
REMARK 4.2. Algorithm 1 works under fairly general conditions, as it only requires two consistent estimators of $\underline{c}$ and $\bar{c}$. The term $n$, which can be interpreted as the discretization step used for approximating the limiting regulated Brownian motion, only needs to be bounded below by $T$. In principle, Algorithm 1 can be applied to any unit root test with null limiting distribution depending on the regulated Brownian motion $B_{c}^{\bar{c}}$.

REMARK 4.3. As is standard with simulation-based tests, the MC $p$-value $p_{n}^{*}$ can be computed with any desired degree of accuracy by generating $B$ (conditionally) independent samples $\left\{X_{T: b}^{*}\right\}$, $b=1, \ldots, B$, and by computing $\mathcal{A D F}_{\alpha: b}^{*}$ as above on each sample. The simulated $p$-value is then computed as $\tilde{p}_{n}^{*}:=B^{-1} \sum_{b=1}^{B} \mathbb{I}\left(\mathcal{A D F}_{\alpha: b}^{*}<\mathcal{A D F} \mathcal{F}_{\alpha}\right)$, and is such that $\tilde{p}_{n}^{*} \stackrel{\text { a.s. }}{\rightarrow} p_{n}^{*}$ as $B \rightarrow \infty$. An asymptotic standard error is given by $\left(\tilde{p}_{n}^{*}\left(1-\tilde{p}_{n}^{*}\right) / B\right)^{1 / 2}$; cf. Hansen $(1996$, p.419).

REMARK 4.4. In Theorem 2, any number of steps $n$ used to construct the process $X_{n}^{*}$ such that $n \geq T$ is admissible. Given that the simulation-based approach is used to retrieve $p$-values from the asymptotic distribution of the test statistics, it appears natural to consider a large number of steps. However, setting $n=T$ generally provides better approximations to the finite sample distribution of the test statistic, see Section 5 below.

REMARK 4.5. The procedure outlined above can be applied in the one bound case as well. In the case of a single lower (upper) bound, it suffices to set $\widehat{\bar{c}}=+\infty(\underline{\widehat{c}}=-\infty)$.

\subsection{Re-coloured simulation-based tests}

Because the limiting distribution of $\mathcal{A D} \mathcal{F}_{\alpha}$ does not depend on serial correlation nuisance parameters, the MC errors $\varepsilon_{t}^{*}$ (see Step (i) of Algorithm 1) are uncorrelated and the MC statistic $\mathcal{A D F}_{\alpha}^{*}$ in Algorithm 1 does not require a correction for serial correlation. However, an improved finite sample approximation in the presence of serially correlated errors might be anticipated from replacing $\mathcal{A D F}_{\alpha}^{*}$ in step (iii) with the analogue, say $\mathcal{A D F}_{\alpha}^{* *}$, of the original $\mathcal{A D F} \mathcal{F}_{\alpha}$ statistic, computed from the OLS regression

$$
\hat{X}_{t}^{*}=\alpha \hat{X}_{t-1}^{*}+\sum_{i=1}^{k} \alpha_{i} \Delta \hat{X}_{t-i}^{*}+e_{t}^{*}
$$

with $\hat{X}_{t}^{*}$ the de-meaned counterpart of $X_{t}^{*}$. The $p$-value in Step (iv) is then computed using the cdf of $\mathcal{A D F}_{\alpha}^{* *}$, say $G_{n}^{* *}()$. The results in Theorem 2 would be unaltered.

In a further attempt to improve finite sample performance in the case of correlated shocks, the basic MC approach outlined above can be extended to include a re-colouring (or sieve) component, 
without altering the large sample theory given in Theorem 2. As in Ferretti and Romo (1996), Chang and Park (2003) and Cavaliere and Taylor (2009), inter alia, this involves re-building stationary serial correlation into the MC innovations. This can be done by using the estimated stationary lag dynamics obtained from fitting the ADF regression

$$
\hat{X}_{t}=\alpha \hat{X}_{t-1}+\sum_{i=1}^{k_{r c}} \alpha_{i} \Delta \hat{X}_{t-i}+\varepsilon_{t, k_{r c}}
$$

where $k_{r c}$ is the lag truncation used for the purposes of re-colouring. Accordingly, with $\hat{\alpha}_{k_{r c}}(z):=$ $1-\sum_{i=1}^{k_{r c}} \hat{\alpha}_{i} z^{i}$, the recursion in Step (ii) of Algorithm 1 can be replaced by the re-coloured recursion

$$
X_{t}^{*}:=\left\{\begin{array}{cc}
\widehat{\bar{c}} & \text { if } X_{t-1}^{*}+n^{-1 / 2} u_{t, k_{r c}}^{*}>\widehat{\bar{c}} \\
\widehat{\widehat{c}} & \text { if } X_{t-1}^{*}+n^{-1 / 2} u_{t, k_{r c}}^{*}<\widehat{\widehat{c}} \\
X_{t-1}^{*}+n^{-1 / 2} u_{t, k_{r c}}^{*} & \text { otherwise }
\end{array}\right.
$$

where $u_{t, k_{r c}}^{*}$ is the re-coloured innovation process defined through the difference equation

$$
\frac{\hat{\alpha}_{k_{r c}}(L)}{\hat{\alpha}_{k_{r c}}(1)} u_{t, k_{r c}}^{*}=\varepsilon_{t}^{*}, t=1, \ldots, T
$$

initialized at $0 .^{2}$ The scheme in (4.13)-(4.14) differs from that in Algorithm 1 in that the estimated AR lag polynomial, $\hat{\alpha}_{k_{r c}}(L)$, is incorporated into the algorithm to re-colour the MC innovations $\varepsilon_{t}^{*}$. Obviously, since the simulated errors are autocorrelated, the $\mathcal{A D F}_{\alpha}^{* *}$ statistic should be considered in step (iii) of the algorithm.

REMARK 4.6. Notice that $k_{r c}$ need not diverge to infinity with the sample size, nor it has to be equal to the truncation lag $k$ used in the original ADF regression, since the re-colouring device is motivated from purely finite sample concerns. The results established in Theorem 2 also apply when re-coloured MC errors are used, provided $k_{r c}=o\left(T^{1 / 2}\right)$.

REMARK 4.7. In small samples there is the possibility that the estimated lag polynomial could have one or more explosive roots. We found that the performance of the algorithm was improved if any such root was shrunk to have modulus less than unity. In our experiments reported in Section 5 below we scaled such estimated roots to have modulus equal to .99 .

\footnotetext{
${ }^{2}$ Notice that the $\hat{\alpha}_{k_{r c}}(1)^{-1}$ factor appearing on the left hand side of (4.14) ensures that $u_{t, k_{r c}}^{*}$ has unit long run variance. This normalization guarantees that $X_{n}^{*}(\cdot):=X_{\lfloor n \cdot\rfloor}^{*}$ converges weakly to $B_{\underline{c}}^{\bar{c}}(\cdot)$ in probability, as required.
} 


\subsection{Extension to heteroskedastic shocks}

Assumption $\mathcal{A}_{1}$ allows for cases where the innovation process $\left\{v_{t}\right\}$ in (2.4) is a (second order) stationary martingale difference sequence. This assumption therefore allows for certain forms of conditional heteroskedasticity. Unconditional heteroskedasticity, as considered by Cavaliere and Taylor $(2007,2008)$, can alter the large sample results given in this paper. Precisely, under the decomposition $v_{t}:=\sigma_{t} z_{t}$ with $z_{t}$ an i.i.d. $(0,1)$ process (with bounded fourth order moment) and $\sigma_{t}$ satisfying $\sigma_{t}:=\omega(t / T)>0$ for all $t=1, \ldots, T$, where $\omega(\cdot) \in \mathcal{D}$ is deterministic, Cavaliere and Taylor (2007) show that $T^{-1 / 2} Y_{\lfloor T \cdot\rfloor}:=T^{-1 / 2} \sum_{t=1}^{\lfloor T \cdot\rfloor} u_{t}=T^{-1 / 2} C(1) \sum_{t=1}^{\lfloor T \cdot\rfloor} \varepsilon_{t}+o_{p}(1) \stackrel{w}{\rightarrow} \lambda_{\omega} M(\cdot)$ where, for $\bar{\omega}:=\left(\int_{0}^{1} \omega^{2}\right)^{1 / 2}, \lambda_{\omega}^{2}:=\bar{\omega}^{2} C(1)^{2}$ and $M$ is the continuous time Martingale $M(\cdot):=$ $\bar{\omega}^{-1} \int_{0}^{\cdot} \omega d B$ ( $B$ being a standard Brownian motion). It can be shown that, in this case, Theorem 1 is no longer appropriate. Rather, the limiting distribution of $T^{-1 / 2} Y_{\lfloor T \cdot\rfloor}$ and of the unit root statistics are as given in Theorem 1 but with the regulated Brownian motion $B_{\underline{c}}^{\bar{c}}$ replaced by a Martingale process, regulated at $\underline{c}$ and $\bar{c}$, say $M_{\underline{c}}^{\bar{c}} \cdot 3$ Consequently, the simulation-based tests earlier proposed are no longer valid, as they do not allow to replicate the time-varying behavior of the unconditional variance of the shocks.

Nevertheless, a simple way of accounting for (possible) unconditional heteroskedasticity can be achieved by using a wild-bootstrap type construction of the simulated innovations, $\varepsilon_{t}^{*}$. Specifically, instead of generating $\varepsilon_{t}^{*}$ as an i.i.d. process, we can set $\varepsilon_{t}^{*}:=\hat{\varepsilon}_{t, k_{r c}} z_{t}$, where $z_{t}$ is an i.i.d. $N(0,1)$ sequence (independent of the original sample) and $\hat{\varepsilon}_{t, k_{r c}}$ are the residuals from the ADF regression (4.12); see Cavaliere and Taylor (2008, 2009). Given the preceding results, it can reasonably be conjectured that the large sample results of Theorem 2 remain valid even under unconditional heteroskedasticity of the type described here. Although a full asymptotic analysis of this case is beyond the scope of this paper, extensive simulation results support this view.

It is worth noting, however, that the type of heteroskedasticity which can be allowed using the wild bootstrap approach does not cover cases where the volatility of the innovations is related to the levels of the process. For instance, EMS target zone exchange rates tend to be more volatile as the exchange rate approaches the bound; conversely, for nominal interest rates volatility is positively related to the levels. Unfortunately, most studies in unit root and co-integration seem to neglect this possible relation between levels and volatility and - to our knowledge - no asymptotic theory is available for these processes in the non-stationary case (for a small class of level-dependent heteroskedastic, but stationary, processes, see e.g. Ling, 2002). Nevertheless, in a Monte Carlo

\footnotetext{
${ }^{3}$ This process can be constructed as a regulated Brownian motion, see Harrison (1985) or Cavaliere (2005), but with the standard Brownian motion replaced by the $M$.
} 
study, Rodrigues and Rubia (2005) show that level-dependent heteroskedasticity does not seem to affect the size of the unit root tests. In the bounded case, a number of Monte Carlo experiments (available from the authors upon request) show that the size of our tests are also only marginally affected by level-dependent heteroskedasticity.

\section{$5 \quad$ Finite sample simulations}

In this section we use MC methods to analyze the finite sample size of the bound-corrected unit root tests of Section 4 for a variety of bounded integrated processes.

Data are generated as in (2.1)-(2.2) for $T=100,500$ under the unit root hypothesis $\alpha=1$, where we set $Y_{0}=\theta=0$ without loss of generality. Both the case of two (symmetric) bounds $(\bar{c}=-\underline{c}=: c>0)$, and a single lower bound $(\bar{c}=\infty,-\underline{c}=: c>0)$ are considered. All experiments are conducted using 10,000 replications and using the rndKMn function of Gauss 9.0. ${ }^{4}$

Following Cavaliere (2005) and Ng and Perron (2001), the errors $v_{t}$ in (2.4) are generated as i.i.d. $N(0,1)$. The (conditional) distribution of $u_{t}=\Delta X_{t}$ is then obtained by reflecting ${ }^{5}$ the distribution of $\varepsilon_{t}:=C(L) v_{t}$ at $\underline{b}-X_{t-1}$ and $\bar{b}-X_{t-1}$. Results for different truncation mechanisms do not alter the result reported in this section.

In Section 5.1, to analyze the effects of the presence of the bounds uncontaminated by serial dependence, we set $C(L)=1$ in (2.4) and, correspondingly, $k=0$ in (3.8). The analysis is then extended in Section 5.2 to allow for weak dependence in $\varepsilon_{t}$. In this case, the number of lags in the spectral AR estimator of the long run variance (3.8) is chosen according to the MAIC lag length selection criterion of $\mathrm{Ng}$ and Perron (2001) with $k \leq\left\lfloor 12(T / 100)^{0.25}\right\rfloor$.

Three different version of the simulation-based unit root tests are employed. In the first version we consider the $A D F^{*}$ and $M^{*}$ tests constructed according to Algorithm 1, where we set the discretization step $n$ to $20,000 .{ }^{6}$ The second version differs from the first since we set $n=T$, so that the simulated test statistic reflects exactly the corresponding length of the original sample. Although the two variants are asymptotically equivalent, we aim at assessing whether using a lower discretization step improves the finite sample size of the test. Finally, in the autocorrelated case we also consider the effect of adding the re-coloured device described in Section 4 to the

\footnotetext{
${ }^{4}$ The Gauss procedure for computing the simulation-based $p$-values is available from the authors upon request, or can be downloaded from the web page http://www2.stat.unibo.it/cavaliere/lts-adf/.

${ }^{5}$ Specifically, we set $\underline{\xi}_{t}:=2\left(\underline{b}-\left(X_{t-1}+u_{t}\right)\right) \mathbb{I}\left(X_{t-1}+u_{t}<\underline{b}\right)$ and $\bar{\xi}_{t}:=2\left(\left(X_{t-1}+u_{t}\right)-\bar{b}\right) \mathbb{I}\left(X_{t-1}+u_{t}>\bar{b}\right)$.

${ }^{6}$ Since the case $n=20,000$ is computationally burdensome, we implemented this algorithm as follows. We applied Algorithm 1 with $n=20,000, B=50,000$ and setting $\widehat{\bar{c}}=-\underline{\widehat{c}}=c(-\underline{\widehat{c}}=c$ in the single bound case), with $c$ taking values on the grid $0.01,0.02, \ldots, 10$. For each $c$, the corresponding critical values were stored. Simulation-based critical values were then retrieved through a linear interpolation of the stored critical values.
} 
algorithm. For all variants, the MC errors $\varepsilon_{t}^{*}$ are $N(0,1)$. Moreover, each test rejects the null hypothesis when the corresponding simulated $p$-value is below the nominal asymptotic $5 \%$ level. $p$-values are computed as in Remark 4.3 with $B=499$. For space constraints, only results for the OLS de-trended statistics are reported.

\subsection{Uncorrelated errors}

Table 1 reports the empirical rejection frequency [ERF], under the null hypothesis $\alpha=1$ in (2.1)(2.2), of the simulation-based $A D F^{*}$ and $M^{*}$ tests of Section 4.2. The tests based on $n=20,000$ are denoted by 'a' while tests based on $n=T$ are denoted by 'b'. In order to evaluate the impact of (neglected) bounds on the size of standard unit root tests, we also report the ERF for the standard $A D F$ and $M$ tests.

\section{[Table 1 about here]}

The upper panel of Table 1 reports the ERF of the various tests in the two-bound case (the case of no bounds corresponds to the ' $\infty$ ' entry), while single bound case is reported in the lower panel.

Consider first the standard $A D F$ and $M$ tests, where the bounds are neglected. In the presence of bounds, they are generally oversized, relative to the benchmark case of no bounds $(c=\infty)$. For instance, the ERFs of the $\mathcal{A D F}_{\alpha}$ and $\mathcal{M Z}_{\alpha}$ tests, which are quite accurate for $c=\infty$ and $T=500$, increase to $30 \%$ and $29 \%$ when there are two bounds with $c=0.4$ and $T=500$. The $\mathcal{A D F}_{t}$ and $\mathcal{M Z}_{t}$ tests are also oversized in the presence of two bounds, with ERF around $20 \%$ in the two-bound case with $c=0.4$ and $T=500$. The $\mathcal{M S B}$ test appears to be the worst affected (its ERF is up to $34 \%$ ). Significant over-sizing can also be observed in the one-bound case, where in the case of a process starting at the lower bound $(c=0)$, most tests have ERFs between $18 \%$ and $21 \%$. These results show that in the presence of bounds, standard unit root tests can over-reject the unit root null hypothesis.

Turning to the simulation-based tests, the results in Table 1 show that the size accuracy of the tests is extremely good. In the two-bounds case, for all values of $c$ considered the $A D F^{*}$ and $M^{*}$ tests based on $n=20,000$ (columns 'a' in the table) are as accurate as the standard $A D F$ and $M$ test in the unbounded case. For instance, in the unbounded case, the ERF of the $\mathcal{A D F}_{t}^{*}$ and the $\mathcal{A D F}_{t}$ tests is only slightly above $5 \%$ for both $T=100$ and $T=500$. Conversely, for $c=0.4$ the ERF of $\mathcal{A D F}_{t}^{*}$ is still about $5 \%$ (specifically, $5.4 \%$ for $T=100$ and $4.7 \%$ for $T=500$ ), whereas the standard $\mathcal{A D} \mathcal{F}_{t}$ test has ERF above $20 \%$ for both $T=100$ and $T=500$. Some of the 
simulation-based tests, in particular the $M^{*}$ tests, appear to be marginally undersized, especially in the one-bound case; however, the overall performance is largely satisfactory, even when the bounds are tight.

Improved size properties are obtained by considering the simulation-based tests with discretization step $n=T$ (columns ' $\mathrm{b}$ ' in the table). Almost all the $A D F^{*}$ and $M^{*}$ tests have ERFs very close to $5 \%$. Even the $M^{*}$ tests, which appear to be slightly undersized for $n=20,000$, have ERFs close to $5 \%$ when $n=T$. The superiority of the $A D F^{*}$ and $M^{*}$ tests with $n=T$ can be observed both in the two-bound and in the one-bound cases. Finally, it is worth noting that the tests based on $n=T$ generally outperform the asymptotic tests discussed in Cavaliere (2005).

\subsection{Autocorrelated errors}

The properties of the $A D F^{*}$ and $M^{*}$ tests under the unit root null hypothesis are now examined for $\varepsilon_{t}$ following a linear process. Two cases are considered. First, $\varepsilon_{t}$ is a stationary $\operatorname{AR}(1)$ process, i.e. $\varepsilon_{t}=\phi \varepsilon_{t-1}+\nu_{t}$, so that $C(L)=\left(1+\phi L+\phi^{2} L^{2}+\ldots\right)$ in (2.4). The term $\nu_{t}$ is i.i.d. $N\left(0,(1-\phi)^{2}\right)$ and the AR parameter $\phi \in\{-0.5,0.5\}$. Second, $\varepsilon_{t}$ is $M A(1)$, i.e. $\varepsilon_{t}=\theta \nu_{t-1}+\nu_{t}$, so that $C(L)=1+\theta L$ in $(2.4)$. In this case $\nu_{t}$ is i.i.d. $N\left(0,1 /\left(1+\theta^{2}\right)\right)$ with $\theta \in\{-0.5 ; 0.5\}$. In all cases the long-run variance of $\left\{\varepsilon_{t}\right\}$ is unity.

Together with the $A D F^{*}$ and $M^{*}$ tests of Section 4.2 with $n=20,000$ and $n=T$, we also report the simulation-based tests employing the re-colouring device of Section 4.3. For the latter tests, the re-colouring lag truncation parameter is $k_{r c}=k .^{7}$

\section{[Tables 2 \& 3 about here]}

Results for the two-bound case are reported in Table 2. In the presence of autocorrelated errors, the standard $A D F$ and $M$ tests are generally oversized. For instance, when $c=0.4$ and $T=500$, $\mathcal{A D F}_{t}$ and $\mathcal{M Z}_{t}$ have ERFs around $13 \%$ for positively autocorrelated errors, and around $30 \%$ for negatively correlated errors. The highest $\operatorname{ERF}(71 \%)$ is obtained through $\mathcal{A D F}_{\alpha}$ with negative MA errors, $c=0.4$, and $T=100$.

With $\operatorname{AR}(1)$ errors, the $A D F^{*}$ and $M^{*}$ tests based on $n=20,000$ (columns 'a' in the table) are slightly undersized for both positive and negative $\phi$, particularly when the bounds are tight (it is worth recalling that in the presence of autocorrelated errors, when there are no bounds the

\footnotetext{
${ }^{7}$ As in Cavaliere and Taylor (2009), in unreported simulations we also set $k_{r c}=4$. The corresponding sizes were quite close to those obtained for $k_{r c}=k$; however, tests based on $k_{r c}=k$ are generally preferable.
} 
automatic data-dependent lag selection rule employed make all unit root tests slightly conservative).

Setting $n=T$ in Algorithm 1 (columns 'b' in the table) improves the finite sample performance of almost all tests, in particular for $T=100$. For instance, when the bounds are very close $(c=0.4), \mathcal{M S B}^{*}$ has ERF $0.2 \%$ for $T=100$ and $\phi=-0.5$, while the ERF increases to $2.5 \%$ when the discretization step is $n=T$. This effect characterizes almost all tests considered.

Massive size improvements are obtained when the re-colouring device is added to Algorithm 1 (columns ' $c$ ' in the table). Now, even for moderate sample sizes, the performance of the tests are largely satisfactory, with almost all tests having ERF close to 5\%, even for small values of $c$. Taking again the case of $\mathcal{M S B}^{*}$ to illustrate, for $T=100, \phi=-0.5$ and $c=0.4$, the ERF grows from $0.2 \%$ to $4 \%$ when the proposed re-colouring device is used.

The results for the case of MA errors are comparable to those obtained for AR errors. The proposed simulation-based tests perform particularly well, in particular when the re-colouring device is used. There is evidence of oversizing for $\phi=-0.5$, but only for $c=0.4$ and $T=100$. Also in this case the re-colouring device basically improves all tests.

The results for one single (lower) bound, reported in Table 3, confirm those obtained for the case of two bounds. The proposed simulation-based tests seem to control size properly even in the presence of autocorrelated disturbances. For some parameter configurations, tests without re-colouring device tend to be undersized. However, tests based on re-colouring appear to be largely satisfactory for all the error processes and all values of the bound parameter $c$ considered.

\section{Empirical illustration}

In this section we illustrate the methods discussed in this paper with a short application to nominal interest rate dynamics. Well-known examples are e.g. econometric models for the term structure (see Campbell and Shiller, 1987, for an early reference), tests of the so-called Fisher hypothesis (Rose, 1988), joint tests of PPP and UIP (Johansen and Juselius, 1992). In this framework, unit root (and cointegration) tests on nominal interest rates have been extensively applied. Despite the existence of a vast literature on the time series properties of nominal interest rates, it is somewhat surprising that most of the papers do not emphasize that nominal interest rates are non-negative and hence cannot be $\mathrm{I}(1)$ in the usual sense ${ }^{8}$. Focusing on tests for a unit root, in this section we shed some light on this issue by explicitly taking the lower bound at zero into account.

\footnotetext{
${ }^{8}$ An exception is Nicolau (2002).
} 
We consider monthly data of 3-month U.S. Treasury Bill (T-bill) rate from January 1957 to September $2008(T=621)$, see Figure 1. Data are obtained from the International Financial Statistics CD-Rom (2008) of the International Monetary Fund.

\section{[Figure $1 \&$ Table 4 about here]}

In the left panel of Table 4, the standard $A D F$ and $M$ tests are reported along with the corresponding (standard) asymptotic $p$-values. For all tests, the lag truncation parameter $k$, selected according to the MAIC criterion of Ng and Perron (2001) with $k \leq\left\lfloor 12(T / 100)^{0.25}\right\rfloor$, equals 16 ; the corresponding estimate of the long run variance is $s_{A R}^{2}(k)=0.63$. All statistics were computed on OLS de-meaned data. Results for pseudo GLS de-meaned data do not differ and are omitted for brevity.

Without exceptions, all standard tests strongly reject the unit root hypothesis. All $p$-values are below $1 \%$, with the strongest rejection obtained from $\mathcal{M Z}_{\alpha}$ and $\mathcal{M S B}$ tests (the corresponding $p$-values are about $0.3 \%)$. Although this result seems to point against the presence of a unit root in nominal interest rates data, it may actually be affected by the fact that the lower bound at zero may actually increase the empirical rejection frequencies of standard unit root tests. This is confirmed by looking at the estimate of the bound parameter $\underline{c}$. Using $\underline{\widehat{c}}$ of Section 4.1 , we obtain $\underline{\widehat{c}}=-0.16$. According to the simulation results in Table $1,{ }^{9}$ the ERF of a nominal $5 \%$ asymptotic test based on $\mathcal{A D F}_{\alpha}$ or $\mathcal{M Z}_{\alpha}$ is not less than $17 \%$ when the bound parameter $\underline{c}$ is -0.20 (or above) and $T=500$. Similarly, the $\mathcal{A D F}_{t}, \mathcal{M Z}_{t}$ and $\mathcal{M S B}$ tests have ERFs exceeding $14 \%, 13 \%$ and $19 \%$, respectively. ${ }^{10}$ Hence, because of the lower bound at zero, on the basis of standard unit root tests it is not possible to assess whether the rejection of the unit root hypothesis is due to the presence of the bound (i.e., the DGP is a bounded unit root process) or whether the rejection should be taken as evidence of no unit roots (i.e., the DGP is a bounded process with no unit roots).

Tests of the unit root hypothesis where the effect of the bounds is properly taken into account can be performed using the simulation-based approach of Section 4. In the right panel of Table 4 we report the simulation-based $p$-values for the $A D F^{*}$ and $M^{*}$ tests of Section 4.2 with $n=20,000$ (column 'a') and $n=T$ (column 'b'). Moreover, we also report the re-coloured simulation-based $p$-values of Section 4.3 (columns 'c'), where we set $k_{r c}=k$.

\footnotetext{
${ }^{9}$ Although the results in Table 1 refer to the case of white noise errors, they still provide a clear indication of the tendency of standard unit root tests to be seriously oversized when there is a lower bound at -0.16 .

${ }^{10}$ This is in agreement with the findings in Cavaliere (2005, figure 5), where for $\underline{c} \in[-0.20,0]$ the asymptotic size of most unit root tests at the nominal $5 \%$ (asymptotic) level is about $20 \%$.
} 
The proposed simulation-based tests reverse the conclusion of standard unit root tests. It can immediately be noticed that all $p$-values become much higher when the lower bound at zero is taken into account. This evidence favours the maintenance of the unit root hypothesis. The tests based on the re-colouring device (the most reliable in finite samples, according to the Monte Carlo experiment in Section 5), have $p$-values in the range 0.08-0.12.

Several points can be made out of this analysis. First of all, the presence of bounds affects the outcome of standard unit root tests, as predicted by the asymptotic theory. This can be immediately seen from the inspection of the $p$-values obtained with and without taking account of the bound: when the bound is considered, all $p$-values increase remarkably. Second, standard unit root tests are not useful for understanding whether the rejection of the unit root null hypothesis should be attributed to the presence of the bound or to the absence of a unit root. In the special case considered in this section, standard unit root tests lead to the conclusion that the interest rate considered is not a unit root process. Conversely, when the bound is accounted for, this conclusion is reversed. Interestingly, this result is not at odd with the conclusions in Aït-Sahalia (1996), who suggests (using high frequency data) that the US interest rate is likely to behave as a unit root process most of the time, but it reverts toward its mean when it reaches low values.

\section{Conclusions}

When applied to bounded time series, conventional unit root tests have to be treated with care. This paper shows that the popular $A D F$ unit root tests as well as the so-called $M$ tests can be unreliable when applied to bounded time series. Specifically, the asymptotic distributions of the corresponding test statistics depend on nuisance parameters related to the position of the bounds; the null distributions are shifted to the left. As a consequence, the rejection of the unit root hypothesis based on standard $p$-values might be due to the fact that the time series of interest is actually bounded.

To rectify this problem, in this paper we discuss a new approach for computing $p$-values (and critical values) for unit root tests in time series which are bounded above, below, or both. Our approach combines the standard $A D F$ and $M$ statistics with a simulation-based approach to constructing the relevant $p$-values. It allows to test statistically whether a bounded time series reverts because of the presence of the bounds alone or because it does not have a unit root. Numerical evidence suggests that our proposed simulation-based procedure works extremely well in finite samples, in particular when it is used in conjunction with a re-colouring device. Moreover, 
the new tests outperforms the Phillips-Perron type tests analyzed in Cavaliere (2005).

Although the class of processes considered here is rather general, some important features of bounded time series are still left aside for future research. Throughout it has been assumed that the bounds are fixed. Hence, cases such as target zone exchange rates under realignments of the central parity are not covered. Nevertheless, our analysis can be generalized to cases of timevarying (known) bounds; see Cavaliere (2000) for linearly trending bounds and Carrion-i-Silvestre and Gadea (2010) for the case of exogenous changes in the bound location. Specifically, most of the results given in this paper continue to hold when the bounds are time varying and satisfy $\left(\underline{b}_{t}-\theta\right) /\left(\lambda T^{1 / 2}\right)=\underline{f}(t / T)+o(1)$ and $\left(\bar{b}_{t}-\theta\right) /\left(\lambda T^{1 / 2}\right)=\bar{f}(t / T)+o(1)$, with $\underline{f} \leq 0$ and $\bar{f} \geq 0$ being càdlàg functions on $[0,1]$.

This paper deals with the (most likely) case that the bounds are known. However, even for unknown bounds the framework developed in this paper can provide useful insights. Where it is known that the time series of interest is regulated, but levels at which regulation occurs are unknown, a reasonable range for the bounds can often be inferred from historical observations and/or from the relevant economic theory. Moreover, by using our approach one can determine a minimum range under which the $A D F$ and $M$ tests do not suffer from oversizing, see Herwartz and $\mathrm{Xu}$ (2008) for the analysis of current account imbalances. The minimum range is defined by 'break even' bounds which approximately equalize the $p$-value of the unit root test considering bounds and the $p$-value obtained ignoring bounds. Unreasonably large break-even bounds signifies that neglecting the bounds when testing for a unit root might be misleading. In addition, it is possible to construct a (conservative) test for the unit root hypothesis by taking the minimum of the simulation-based $p$-values over a grid of admissible bound locations.

It is worth emphasizing that only a constant deterministic term is allowed in the paper and extension to more general deterministic components is not straightforward. For instance, the presence of a linear trend has strong implications for bounded variables, as the trend might imply that, as $T$ increases, the series is absorbed at one of the bounds or, in the one-bound case, that it drifts away from the bound (with the latter becoming irrelevant). Given our main assumption that the location of the bounds is related to $T^{1 / 2}$, local linear trends of the form $\theta_{t}=\theta+\tau t$ with $\tau:=\kappa T^{-1 / 2}(\kappa$ being a fixed constant) represent a reasonable solution to introduce linear trends in bounded time series. Alternatively, piecewise-constant deterministic terms can also be considered, see Carrion-i-Silvestre and Gadea (2010). Both extensions are not trivial and beyond the scope of the present paper. 
Finally, given that all the results discussed here hold for univariate time series only, an important and necessary extension is to generalize the proposed simulation-based tests to the case of multiple time series and co-integration tests. Suggestions for this step - currently under investigation by the authors - are given in Granger (2010).

\section{References}

Aït-Sahalia, Y., 1996. Testing Continuous-Time Models of the Spot Interest Rate. Review of Financial Studies 9, 385-426.

Berk, K.N., 1974. Consistent Autoregressive Spectral Estimates. Annals of Statistics 2, 489-502.

Campbell, J.Y. and Shiller, R.J., 1987. Cointegration and Tests of Present Value Models. Journal of Political Economy 95, 1062-1088.

Carrion-i-Silvestre and Gadea, M.D., 2010. Bounds, Breaks and Unit Root Tests. Working paper, University of Barcelona.

Cavaliere, G., 2000. A Rescaled Range Statistics Approach to Unit Root Tests, Econometric Society World Congress 2000 Contributed Papers 0318.

Cavaliere, G., 2005. Limited Time Series With A Unit Root. Econometric Theory 21. 907-945.

Cavaliere, G. and Taylor, A.M.R, 2007. Testing for Unit Roots in Time Series Models with Non-Stationary Volatility. Journal of Econometrics 140, 919-947.

Cavaliere, G. and Taylor, A.M.R, 2008. Bootstrap Unit Root Tests for Time Series Models with Non-Stationary Volatility. Econometric Theory 24, 43-71.

Cavaliere, G. and Taylor, A. M. R., 2009. Heteroskedastic Time Series with a Unit Root. Econometric Theory 25, 1228-1276.

Chang, Y. and Park, J.Y., 2002. On the Asymptotics of ADF Tests for Unit Roots. Econometric Reviews 21, 431-447.

Chang, Y. and Park, J.Y., 2003. A Sieve Bootstrap for the Test of a Unit Root. Journal of Time Series Analysis 24, 379-400.

Dickey, D.A. and Fuller, W.A., 1979. Distribution of the Estimators for Autoregressive Time Series with a Unit Root. Journal of the American Statistical Association 74, 427-431. 
Elliott, G., Rothenberg, T.J. and Stock, J.H., 1996. Efficient Tests for an Autoregressive Unit Root. Econometrica 64, 813-836.

Ferretti, N. and Romo, J., 1996. Bootstrap Tests for Unit Root AR(1) Models. Biometrika 84, 849-860.

Granger, C.W.J., 2010. Some Thoughts on the Development of Cointegration. Journal of Econometrics 158, 3-6.

Hansen, B. E., 1996. Inference When a Nuisance Parameter Is Not Identified under the Null Hypothesis. Econometrica 64, 413-430.

Hansen, B.E. (2000). Testing for Structural Change in Conditional Models. Journal of Econometrics $97,93-115$.

Harrison, J.M., 1985. Brownian Motion and Stochastic Flow Systems. John Wiley \& Sons.

Herwatz H. and Xu, F. 2008. Reviewing the Sustainability/Stationarity of Current Account Imbalances with Tests for Bounded Integration, Manchester School 76, 267-278.

Johansen, S. and Juselius, K., 1992. Testing Structural Hypotheses in a Multivariate Cointegration Analysis of the PPP and the UIP for UK. Journal of Econometrics 53, 211-244.

Lewis, R. and Reinsel, G.C., 1985. Prediction of Multivariate Time Series by Autoregressive Model Fitting. Journal of Multivariate Analysis 16, 393-411.

Müller, U.K. and Elliott, G., 2003. Tests for Unit Roots and the Initial Condition. Econometrica $71,1269-1286$.

Ng, S. and Perron, P., 1995. Unit Root Tests in ARMA Models with Data Dependent Methods for the Selection of the Truncation lag. Journal of the America Statistical Association 90, 268-281.

Ng, S. and Perron, P., 2001. LAG Length Selection and the Construction of Unit Root Tests with Good Size and Power. Econometrica 69, 1519-1554.

Nicolau, J., 2002. Stationary Processes that Look Like Random Walks - The Bounded Random Walk Process in Discrete and Continuous Time. Econometric Theory 18, 99-118.

Perron, P. and Ng, S., 1996. Useful Modifications to some Unit Root Tests with Dependent Errors and their Local Asymptotic Properties. Review of Economic Studies 63, 435-463. 
Phillips, P.C.B, and Perron, P., 1988. Testing for a Unit Root in Time Series Regressions. Biometrika 75, 335-346.

Phillips, P.C.B, and Solo, V., 1992. Asymptotics for Linear Processes. Annals of Statistics 20, 971-1001.

Rodrigues, P.M.M. and Rubia, A., 2005. The Performance of Unit Root Tests under LevelDependent Heteroskedasticity. Economics Letters 89, 262-268.

Rose, A. K., 1988. Is the Real Interest Rate Stable? Journal of Finance 43, 1095-1112.

Said, E.S. and Dickey, D.A., 1984. Testing for Unit Roots in ARMA $(p, q)$ Models with Unknown $p$ and $q$. Biometrika 71, 599-607.

Schmidt, P. and Phillips, P.C.B. 1992. LM Test for a Unit Root in the Presence of Deterministic Trends, Oxford Bulletin of Economics and Statistics 54, 257-287.

Stock, J. H., 1999. A Class of Tests for Integration and Cointegration, in R. Engle and H. White (eds.), Cointegration, Causality and Forecasting: A Festschrift for Clive W.J. Granger, ch. 6, Oxford University Press.

\section{A Appendix}

This appendix is organized as follows. Section A.1 introduces some preliminary asymptotic results. Section A.2 contains the proofs of Theorem 1 and of the related lemmas. Section A.3 reports the proofs of the simulation-based results of Theorem 2 .

\section{A.1 Preliminary Lemmata}

Let $v_{t}^{*}:=v_{t}+\underline{\xi}_{t}^{*}-\bar{\xi}_{t}^{*}$ and $w_{t}^{*}:=\sum_{i=1}^{t} v_{i}^{*}$, with $\underline{\xi}_{t}^{*}, \bar{\xi}_{t}^{*}$ as defined in Section 2. Furthermore, let $r_{t}^{*}:=\underline{\xi}_{t}^{*}-\bar{\xi}_{t}^{*}$. The following results hold as $T$ diverges.

Lemma A.1 Under the assumptions of Theorem $1,\left(\sigma^{2} T\right)^{-1 / 2} w_{\lfloor T \cdot\rfloor}^{*} \stackrel{w}{\rightarrow} B_{\underline{c}}^{\bar{c}}(\cdot)$, in $\mathcal{D}$.

Proof. By the BN decomposition of Remark 2.2,

$$
T^{-1 / 2} \sum_{t=1}^{\lfloor T \cdot\rfloor} u_{t}=C(1) T^{-1 / 2} \sum_{t=1}^{\lfloor T \cdot\rfloor} v_{t}^{*}+T^{-1 / 2} \tilde{u}_{0}-T^{-1 / 2} \tilde{u}_{\lfloor T \cdot\rfloor}
$$


with $\tilde{u}_{t}=\sum_{j=0}^{\infty} \tilde{c}_{j} v_{t-j}^{*}\left(\tilde{c}_{j}:=\sum_{i=j+1}^{\infty} c_{i}\right)$. Since, under Assumptions $\mathcal{A}$ and $\mathcal{B}, \sup _{t}\left|\tilde{u}_{t}\right|=o_{p}\left(T^{1 / 2}\right)$, we have that $T^{-1 / 2} \sum_{t=1}^{\lfloor T \cdot\rfloor} u_{t}-C(1) T^{-1 / 2} \sum_{t=1}^{\lfloor T \cdot\rfloor} v_{t}^{*} \stackrel{p}{\rightarrow} 0$. From Theorem 1 in Cavaliere (2005) it holds that $\left(\lambda^{2} T\right)^{-1 / 2} \sum_{t=1}^{\lfloor T \cdot\rfloor} u_{t} \stackrel{w}{\rightarrow} B_{\underline{c}}^{\bar{c}}(\cdot)$. Hence, $\lambda^{-1} C(1) T^{-1 / 2} \sum_{t=1}^{\lfloor T \cdot\rfloor} v_{t}^{*} \stackrel{w}{\rightarrow} B_{\underline{c}}^{\bar{c}}(\cdot)$. The proof is completed by noticing that $\lambda^{-1} C(1)=\sigma^{-1}$.

Lemma A.2 Under the assumptions of Theorem $1, T^{-1} \sum_{t=1}^{T} v_{t}^{* 2} \stackrel{p}{\rightarrow} \sigma^{2}$.

ProOF. First, notice that

$$
\frac{1}{T} \sum_{t=1}^{T} v_{t}^{* 2}=\frac{1}{T} \sum_{t=1}^{T}\left(v_{t}+r_{t}^{*}\right)^{2}=\frac{1}{T} \sum_{t=1}^{T} v_{t}^{2}+\frac{1}{T} \sum_{t=1}^{T}\left(r_{t}^{* 2}+2 v_{t} r_{t}^{*}\right)
$$

where $T^{-1} \sum_{t=1}^{T} v_{t}^{2} \stackrel{p}{\rightarrow} \sigma^{2}$ by Assumption $\mathcal{A}_{1}(b)$ and $T^{-1} \sum_{t=1}^{T}\left(r_{t}^{* 2}+2 v_{t} r_{t}^{*}\right)=o_{p}(1)$. To show the latter result it suffices to notice that

$$
\left|\frac{1}{T} \sum_{t=1}^{T}\left(r_{t}^{* 2}+2 v_{t} r_{t}^{*}\right)\right| \leq \frac{1}{T} \sum_{t=1}^{T}\left|r_{t}^{* 2}+2 v_{t} r_{t}^{*}\right| \leq \frac{1}{T}\left\{\max \left|2 v_{t}\right|+\max \left|r_{t}^{*}\right|\right\} \sum_{t=1}^{T} 2\left|r_{t}^{*}\right|=o_{p}(1)
$$

as $\max \left|v_{t}\right|, \max \left|r_{t}^{*}\right|$ are of $o_{p}\left(T^{1 / 2}\right)$, and $\sum_{t=1}^{T}\left|r_{t}^{*}\right|=O_{p}\left(T^{1 / 2}\right)$ under Assumption $\mathcal{B}$.

Lemma A.3 Under the assumptions of Theorem 1, $T^{-1 / 2} \sum_{t=1}^{T} r_{t}^{*}=O_{p}(1)$.

Proof. It follows as $T^{-1 / 2} \sum_{t=1}^{T} r_{t}^{*}=T^{-1 / 2} \sum_{t=1}^{T} v_{t}^{*}-T^{-1 / 2} \sum_{t=1}^{T} v_{t}=O_{p}(1)$ according to Lemma A.1 and a standard FCLT (Phillips and Solo, 1992).

\section{A.2 Proof of Theorem 1 and related Lemmas}

Proof of TheOrem 1. Throughout, to simplify the proof it is assumed (without loss of generality) that $v_{t}=\underline{\xi}_{t}^{*}=\bar{\xi}_{t}^{*}=0$ for all $t \leq 0$ and that no deterministics are included in the model and in the estimation. Furthermore, we sketch the proof for the $\mathcal{A D F} \mathcal{F}_{t}$ and $\mathcal{A D F} \mathcal{F}_{\alpha}$ statistics; results for the other statistics follow similarly.

Let $Z_{t, k}:=\left(\Delta X_{t-1}, \ldots, \Delta X_{t-k}\right)^{\prime}, \beta:=\left(\alpha_{1}, \ldots, \alpha_{k}\right)^{\prime}$ and recall that the ADF regression is

$$
X_{t}=\alpha X_{t-1}+\beta^{\prime} Z_{t, k}+v_{t, k}
$$

with $v_{t, k}=v_{t}^{*}+\sum_{i=k+1}^{\infty} \alpha_{i} \Delta X_{t-i}=v_{t}^{*}+\sum_{i=k+1}^{\infty} \alpha_{i} u_{t-i}$. The proof for $\mathcal{A D F}_{t}$ is as follows. First, under the null hypothesis $\alpha=1$, using Lemma A.2 and Lemmas A.4, A.5 below, we can proceed 
as in Chang and Park (2002) to prove that

$$
\mathcal{A D F}_{t}=\sigma^{-1}\left(T^{-2} \sum_{t=1}^{T} w_{t-1}^{* 2}\right)^{-1 / 2}\left(T^{-1} \sum_{t=1}^{T} w_{t-1}^{*} v_{t}^{*}\right)+o_{p}(1)
$$

with $w_{t}^{*}$ and $v_{t}^{*}$ as previously defined. Then, Lemma A.1, the continuous mapping theorem [CMT] and Lemma A.2 imply

$$
\begin{aligned}
& T^{-1} \sum_{t=1}^{T} w_{t-1}^{*} v_{t}^{*}=\frac{1}{2 T} w_{T}^{* 2}-\frac{1}{2 T} \sum_{t=1}^{T} v_{t}^{* 2} \stackrel{w}{\rightarrow} \frac{\sigma^{2}}{2}\left(B_{\underline{c}}^{\bar{c}}(1)^{2}-1\right) \\
& T^{-2} \sum_{t=1}^{T} w_{t-1}^{* 2} \stackrel{w}{\rightarrow} \sigma^{2} \int_{0}^{1} B_{\underline{c}}^{\bar{c}}(s)^{2} d s
\end{aligned}
$$

which completes the proof for $\mathcal{A D F}_{t}$. Similarly,

$$
\mathcal{A} \mathcal{D} \mathcal{F}_{\alpha}=\frac{T(\hat{\alpha}-1)}{\hat{\alpha}(1)}=\left(T^{-2} \sum_{t=1}^{T} w_{t-1}^{* 2}\right)^{-1}\left(T^{-1} \sum_{t=1}^{T} w_{t-1}^{*} v_{t}^{*}\right)+o_{p}(1)
$$

Lemma A.5 then implies the consistency of $\hat{\alpha}(1)$, which completes the proof of the above equation.

Lemma A.4 Under the assumptions of Theorem 1, (a) $T^{-1} \sum_{t=1}^{T} X_{t-1} v_{t, k}=C(1) T^{-1} \sum_{t=1}^{T} w_{t-1}^{*} v_{t}^{*}+$ $o_{p}(1)$; (b) $T^{-2} \sum_{t=1}^{T} X_{t-1}^{2}=C(1)^{2} T^{-2} \sum_{t=1}^{T} w_{t-1}^{*}{ }^{2}+o_{p}(1)$; (c) $T^{-1} \sum_{t=1}^{T} v_{t, k}^{2}=T^{-1} \sum_{t=1}^{T} v_{t}^{* 2}+$ $o_{p}(1)$.

Proof. Part (a). We have that

$$
\begin{aligned}
\sum_{t=1}^{T} X_{t-1} v_{t, k}= & \sum_{t=1}^{T} X_{t-1} v_{t}^{*}+\sum_{t=1}^{T} X_{t-1}\left(v_{t, k}-v_{t}^{*}\right) \\
= & \sum_{t=1}^{T}\left(C(1) w_{t-1}^{*}+\tilde{u}_{0}-\tilde{u}_{t-1}\right) v_{t}^{*}+\sum_{t=1}^{T}\left(C(1) w_{t-1}^{*}+\tilde{u}_{0}-\tilde{u}_{t-1}\right)\left(v_{t, k}-v_{t}^{*}\right) \\
= & C(1) \sum_{t=1}^{T} w_{t-1}^{*} v_{t}^{*}+\sum_{t=1}^{T} \tilde{u}_{0} v_{t}^{*}-\sum_{t=1}^{T} \tilde{u}_{t-1} v_{t}^{*}+ \\
& C(1) \sum_{t=1}^{T} w_{t-1}^{*}\left(v_{t, k}-v_{t}^{*}\right)+\sum_{t=1}^{T} \tilde{u}_{0}\left(v_{t, k}-v_{t}^{*}\right)-\sum_{t=1}^{T} \tilde{u}_{t-1}\left(v_{t, k}-v_{t}^{*}\right) \\
= & C(1) \sum_{t=1}^{T} w_{t-1}^{*} v_{t}^{*}+R_{1}+R_{2}+R_{3}+R_{4}+R_{5} .
\end{aligned}
$$

By showing $R_{1}=O_{p}\left(T^{1 / 2}\right)$ and $R_{2}+R_{3}+R_{4}+R_{5}=o_{p}(T)$, the statement (a) follows.

First, $R_{1}=\sum_{t=1}^{T} \tilde{u}_{0} v_{t}^{*}=\tilde{u}_{0}\left(\sum_{t=1}^{T} v_{t}+\sum_{t=1}^{T} r_{t}^{*}\right)=O_{p}\left(T^{1 / 2}\right)$ due to Lemma A.3 and a standard 
FCLT. Second,

$$
\begin{aligned}
R_{2} & =\sum_{t=1}^{T} \tilde{u}_{t-1} v_{t}^{*}=\sum_{t=1}^{T} \sum_{j=0}^{\infty} \tilde{c}_{j} v_{t-1-j}^{*} v_{t}^{*}=\sum_{t=1}^{T} \sum_{j=0}^{\infty} \tilde{c}_{j}\left(v_{t-1-j}+r_{t-1-j}^{*}\right)\left(v_{t}+r_{t}^{*}\right) \\
& =\sum_{t=1}^{T} \sum_{j=0}^{\infty} \tilde{c}_{j} v_{t-1-j} v_{t}+\sum_{t=1}^{T} \sum_{j=0}^{\infty} \tilde{c}_{j}\left(v_{t-1-j} r_{t}^{*}+r_{t-1-j}^{*} v_{t}+r_{t-1-j}^{*} r_{t}^{*}\right)=o_{p}(T) .
\end{aligned}
$$

Since $\sum_{j=0}^{\infty} \tilde{c}_{j} v_{t-1-j} v_{t}$ is a MDS with finite variance, it can be shown that $\sum_{t=1}^{T} \sum_{j=0}^{\infty} \tilde{c}_{j} v_{t-1-j} v_{t}=$ $o_{p}(T)$. Also,

$$
\begin{aligned}
& \left|\sum_{t=1}^{T} \sum_{j=0}^{\infty} \tilde{c}_{j}\left(v_{t-1-j} r_{t}^{*}+r_{t-1-j}^{*} v_{t}+r_{t-1-j}^{*} r_{t}^{*}\right)\right| \leq \sum_{t=1}^{T} \sum_{j=0}^{\infty}\left|\tilde{c}_{j}\right|\left|v_{t-1-j} r_{t}^{*}+r_{t-1-j}^{*} v_{t}+r_{t-1-j}^{*} r_{t}^{*}\right| \\
\leq & \sum_{t=1}^{T} \sum_{j=0}^{\infty}\left|\tilde{c}_{j}\right| 3\left|r_{t}^{*}\right|\left\{\max \left|v_{t}\right|+\max \left|r_{t}^{*}\right|\right\}=\sum_{j=0}^{\infty}\left|\tilde{c}_{j}\right|\left\{\max \left|v_{t}\right|+\max \left|r_{t}^{*}\right|\right\} \sum_{t=1}^{T} 3\left|r_{t}^{*}\right|=o_{p}(T)
\end{aligned}
$$

since $\max \left|v_{t}\right|$ and $\max \left|r_{t}^{*}\right|$ are of $o_{p}\left(T^{1 / 2}\right), \sum_{j=0}^{\infty}\left|\tilde{c}_{j}\right|<\infty$ and $\sum_{t=1}^{T}\left|r_{t}^{*}\right|=O_{p}\left(T^{1 / 2}\right)$.

Before investigating $R_{3}$, consider first $v_{t, k}-v_{t}^{*}=\sum_{j=k+1}^{\infty} \alpha_{j} u_{t-j}=\sum_{j=k+1}^{\infty} c_{k, j} v_{t-j}^{*}$, where $\sum_{j=k+1}^{\infty} c_{k, j}^{2} \leq a \sum_{j=k+1}^{\infty} \alpha_{j}^{2}=o\left(k^{-2 s}\right)$. Then we have that

$$
\begin{aligned}
R_{3} & =\sum_{t=1}^{T} w_{t-1}^{*}\left(v_{t, k}-v_{t}^{*}\right)=\sum_{t=1}^{T} \sum_{i=1}^{t-1} v_{i}^{*} \sum_{j=k+1}^{\infty} c_{k, j} v_{t-j}^{*}=\sum_{j=k+1}^{\infty} c_{k, j} \sum_{t=1}^{T} v_{t-j}^{*} \sum_{i=1}^{t-1} v_{i}^{*} \\
& =o\left(k^{-s}\right) O_{p}\left(T^{1 / 2}\right) O_{p}\left(T^{1 / 2}\right)=o_{p}\left(T k^{-s}\right)
\end{aligned}
$$

given Assumption $\mathcal{A}_{2}$ and Lemma A.1. Next,

$$
\begin{aligned}
R_{4} & =\sum_{t=1}^{T} \tilde{u}_{0}\left(v_{t, k}-v_{t}^{*}\right)=\sum_{t=1}^{T} \tilde{u}_{0} \sum_{j=k+1}^{\infty} c_{k, j} v_{t-j}^{*}=\tilde{u}_{0} \sum_{j=k+1}^{\infty} c_{k, j} \sum_{t=1}^{T} v_{t-j}^{*} \\
& =o\left(k^{-s}\right) O_{p}\left(T^{1 / 2}\right)=o_{p}\left(T^{1 / 2} k^{-s}\right) \\
R_{5} & =\sum_{t=1}^{T} \tilde{u}_{t-1}\left(v_{t, k}-v_{t}^{*}\right)=\sum_{t=1}^{T}\left(\sum_{j=0}^{\infty} \tilde{c}_{j} v_{t-1-j}^{*}\right)\left(\sum_{j=K+1}^{\infty} c_{k, j} v_{t-j}^{*}\right) \\
& =\sum_{j=0}^{\infty} \sum_{j=k+1}^{\infty} \tilde{c}_{j} c_{k, j} \sum_{t=1}^{T} v_{t-1-j}^{*} v_{t-j}^{*},
\end{aligned}
$$


where

$$
\begin{aligned}
\sum_{t=1}^{T} v_{t-1-j}^{*} v_{t-j}^{*} & =\sum_{t=1}^{T}\left(v_{t-1-j}+r_{t-1-j}^{*}\right)\left(v_{t-j}+r_{t-j}^{*}\right) \\
& =\sum_{t=1}^{T}\left(v_{t-1-j} v_{t-j}\right)+\sum_{t=1}^{T}\left(v_{t-1-j} r_{t-j}^{*}+r_{t-1-j}^{*} v_{t-j}+r_{t-1-j}^{*} r_{t-j}^{*}\right)=o_{p}(T)
\end{aligned}
$$

since $\sum_{t=1}^{T} v_{t-1-j} v_{t-j}=o_{p}(T)$ as $v_{t-1-j} v_{t-j}$ is a MDS with finite variance. Besides, $\sum_{t=1}^{T}\left(v_{t-1-j} r_{t-j}^{*}+\right.$ $\left.r_{t-1-j}^{*} v_{t-j}+r_{t-1-j}^{*} r_{t-j}^{*}\right)=o_{p}(T)$ according to similar arguments as those for $R_{2}$. Furthermore,

$$
\left|\sum_{j=0}^{\infty} \sum_{j=k+1}^{\infty} \tilde{c}_{j} c_{k, j}\right| \leq \sum_{j=0}^{\infty} \sum_{j=k+1}^{\infty}\left|\tilde{c}_{j} c_{k, j}\right|=\sum_{j=0}^{\infty}\left|\tilde{c}_{j}\right| \sum_{j=k+1}^{\infty}\left|c_{k, j}\right|=o\left(k^{-s}\right) .
$$

Therefore, $R_{5}=o_{p}\left(T k^{-s}\right)$. The proof of statement (a) is then complete.

Part (b). According to the BN representation,

$$
\begin{aligned}
\frac{1}{T^{2}} \sum_{t=1}^{T} X_{t-1}^{2} & =\frac{1}{T^{2}} \sum_{t=1}^{T}\left(C(1) w_{t-1}^{*}+\tilde{u}_{0}-\tilde{u}_{t-1}\right)^{2} \\
& =C(1)^{2} \frac{1}{T^{2}} \sum_{t=1}^{T} w_{t-1}^{* 2}+\frac{1}{T^{2}} \sum_{t=1}^{T}\left(\tilde{u}_{0}^{2}-\tilde{u}_{t-1}^{2}-2 \tilde{u}_{0} \tilde{u}_{t-1}+2 C(1) w_{t-1}^{*} \tilde{u}_{0}-2 C(1) w_{t-1}^{*} \tilde{u}_{t-1}\right) \\
& =C(1)^{2} \frac{1}{T^{2}} \sum_{t=1}^{T} w_{t-1}^{* 2}+o_{p}(1),
\end{aligned}
$$

since $\tilde{u}_{t-1}=o_{p}\left(T^{1 / 2}\right)$ and $w_{t-1}^{*}=O_{p}\left(T^{1 / 2}\right)$ as shown in Lemma A.1.

Part (c). The result can be obtained by applying

$$
\left|\left(\frac{1}{T} \sum_{t=1}^{T} v_{t, k}^{2}\right)^{1 / 2}-\left(\frac{1}{T} \sum_{t=1}^{T} v_{t}^{* 2}\right)^{1 / 2}\right| \leq\left[\frac{1}{T} \sum_{t=1}^{T}\left(v_{t, k}-v_{t}^{*}\right)^{2}\right]^{1 / 2} .
$$

The right hand side of the previous inequality satisfies

$$
\begin{aligned}
E\left[\frac{1}{T} \sum_{t=1}^{T}\left(v_{t, k}-v_{t}^{*}\right)^{2}\right] & =E\left[\frac{1}{T} \sum_{t=1}^{T}\left(\sum_{j=k+1}^{\infty} c_{k, j} v_{t-j}^{*}\right)^{2}\right]=E\left[\frac{1}{T} \sum_{t=1}^{T}\left(\sum_{j=k+1}^{\infty} c_{k, j}\left(v_{t-j}+r_{t-j}^{*}\right)\right)^{2}\right] \\
& =E\left[\frac{1}{T} \sum_{t=1}^{T}\left(\sum_{i=k+1}^{\infty} \sum_{j=k+1}^{\infty} c_{k, i} c_{k, j}\left(v_{t-i} v_{t-j}+r_{t-i}^{*} r_{t-j}^{*}+2 v_{t-i} r_{t-j}^{*}\right)\right)\right] \\
& \leq \frac{1}{T} \sum_{t=1}^{T}\left(E\left(v_{t-i} v_{t-j}\right)+\sup \left\{E\left(r_{t-i}^{*} r_{t-j}^{*}\right)+2 E\left(v_{t-i} r_{t-j}^{*}\right)\right\}\right) \sum_{i=k+1}^{\infty} \sum_{j=k+1}^{\infty} c_{k, i} c_{k, j}
\end{aligned}
$$


Note that $\sum_{i=k+1}^{\infty} \sum_{j=k+1}^{\infty} c_{k, i} c_{k, j}=\left(\sum_{j=k+1}^{\infty} c_{k, j}\right)^{2}=o\left(k^{-2 s}\right)$. Furthermore, $E\left(v_{t-i} v_{t-j}\right)+$ $\sup \left\{E\left(r_{t-i}^{*} r_{t-j}^{*}\right)+2 E\left(v_{t-i} r_{t-j}^{*}\right)\right\}<\infty$ due to the stated moment conditions on $\varepsilon_{t}^{*}$ and $r_{t}^{*}$. Therefore, $E\left[T^{-1} \sum_{t=1}^{T}\left(v_{t, k}-v_{t}^{*}\right)^{2}\right]=o\left(k^{-2 s}\right)$, and $T^{-1} \sum_{t=1}^{T}\left(v_{t, k}-v_{t}^{*}\right)^{2}=o_{p}\left(k^{-2 s}\right)$. The statement (c) is then proved.

Lemma A.5 Under the assumptions of Theorem 1, as $T \rightarrow \infty$, (a) $\left\|\left(T^{-1} \sum_{t=1}^{T} Z_{t, k} Z_{t, k}^{\prime}\right)^{-1}\right\|=$ $O_{p}(1)$; (b) $\left\|\sum_{t=1}^{T} Z_{t, k} X_{t-1}\right\|=O_{p}\left(T k^{1 / 2}\right) ;(c)\left\|T^{-1} \sum_{t=1}^{T} Z_{t, k} v_{t, k}\right\|=o_{p}\left(k^{-1 / 2}\right)$.

Proof. Part (a). Let $\gamma_{i}:=E\left(\varepsilon_{t} \varepsilon_{t-i}\right)$ and $\Gamma_{k}:=\left(\gamma_{i-j}\right)_{i, j=1}^{k}$. Denoting $T^{-1} \sum_{t=1}^{T} Z_{t, k} Z_{t, k}^{\prime}$ as $\hat{\Gamma}_{k}$, we have $\left\|\hat{\Gamma}_{k}^{-1}\right\| \leq\left\|\hat{\Gamma}_{k}^{-1}-\Gamma_{k}^{-1}\right\|+\left\|\Gamma_{k}^{-1}\right\|$. Note $\left\|\Gamma_{k}^{-1}\right\|$ is uniformly bounded above by a positive constant $F$ for all $k$ (see e.g. equation (2.14) in Berk (1974)). As the next step, we show that $\left\|\hat{\Gamma}_{k}^{-1}-\Gamma_{k}^{-1}\right\|=o_{p}(1)$ under Assumption $\mathcal{K}$, which completes the statement. First, defining $\epsilon_{t, k}:=\left(\varepsilon_{t-1}, \ldots, \varepsilon_{t-k}\right)^{\prime}$ and $R_{t, k}:=\left(r_{t-1}, \ldots, r_{t-k}\right)^{\prime}$ with $r_{t}=\underline{\xi}_{t}-\bar{\xi}_{t}$, we have

$$
\left\|\hat{\Gamma}_{k}-\Gamma_{k}\right\| \leq\left\|T^{-1} \sum_{t=1}^{T} \epsilon_{t, k} \epsilon_{t, k}^{\prime}-\Gamma_{k}\right\|+\left\|T^{-1} \sum_{t=1}^{T}\left(\epsilon_{t, k} R_{t, k}^{\prime}+R_{t, k} \epsilon_{t, k}^{\prime}+R_{t, k} R_{t, k}^{\prime}\right)\right\|=o_{p}(1) .
$$

Since $E\left(\left\|T^{-1} \sum_{t=1}^{T} \epsilon_{t, k} \epsilon_{t, k}^{\prime}-\Gamma_{k}\right\|\right)^{2} \leq$ constant $k^{2} /(T-k)$ as can be seen e.g. from equations (2.10) and (2.11) in Berk (1974), $\left\|T^{-1} \sum_{t=1}^{T} \epsilon_{t, k} \epsilon_{t, k}^{\prime}-\Gamma_{k}\right\|=O_{p}\left(k / T^{1 / 2}\right)=o_{p}(1)$ under Assumption $\mathcal{K}$. Second, $T^{-1} \sum_{t=1}^{T} \varepsilon_{t-i} r_{t-j}$ and $T^{-1} \sum_{t=1}^{T} r_{t-i} r_{t-j}$ are both of $O_{p}\left(T^{-1 / 2}\right)$. Taking $\sum_{t=1}^{T} \varepsilon_{t-i} r_{t-j}$ to illustrate, we have that for any $a>0, P\left(\left|\sum_{t=1}^{T} \varepsilon_{t-i} r_{t-j}\right|>a\right) \leq a^{-1} H_{T} \sup _{t, i, j} E\left|\varepsilon_{t-i} r_{t-j}\right|$, where $H_{T}$ is the number of times when $r_{t-j}$ is non zero. Since $\sup E\left|\varepsilon_{t-i} r_{t-j}\right|$ is finite under $\mathcal{A}_{1}$ and $\mathcal{B}_{1}$, the desired result holds as $H_{T}=O\left(T^{1 / 2}\right)$ (this follows by the FCLT on the regulators). Hence,

$$
\left\|T^{-1} \sum_{t=1}^{T}\left(\epsilon_{t, k} R_{t, k}^{\prime}+R_{t, k} \epsilon_{t, k}^{\prime}+R_{t, k} R_{t, k}^{\prime}\right)\right\| \leq \sqrt{k^{2}\left(O_{p}\left(T^{-1 / 2}\right)^{2}\right.}=O_{p}\left(k / T^{1 / 2}\right)=o_{p}(1)
$$

under Assumption $\mathcal{K}$. Then, from

$$
\left\|\hat{\Gamma}_{k}^{-1}-\Gamma_{k}^{-1}\right\|=\left\|\hat{\Gamma}_{k}^{-1}\left(\Gamma_{k}-\hat{\Gamma}_{k}\right) \Gamma_{k}^{-1}\right\| \leq\left\|\hat{\Gamma}_{k}^{-1}\right\|\left\|\Gamma_{k}-\hat{\Gamma}_{k}\right\|\left\|\Gamma_{k}^{-1}\right\| \leq\left(\left\|\hat{\Gamma}_{k}^{-1}-\Gamma_{k}^{-1}\right\|+F\right)\left\|\Gamma_{k}-\hat{\Gamma}_{k}\right\| F,
$$

we have $M_{k, T} \leq\left\|\Gamma_{k}-\hat{\Gamma}_{k}\right\|=o_{p}(1)$ with $M_{k, T}:=\left\|\hat{\Gamma}_{k}^{-1}-\Gamma_{k}^{-1}\right\| /\left(\left\|\hat{\Gamma}_{k}^{-1}-\Gamma_{k}^{-1}\right\|+F\right) F$. Thus, $\left\|\hat{\Gamma}_{k}^{-1}-\Gamma_{k}^{-1}\right\|=F^{2} M_{k, T} /\left(1-F M_{k, T}\right)=o_{p}(1)$, which completes the statement (a).

Part (b). To show this statement we can use exactly the same arguments for Lemma 3.2 (b) in 
Chang and Park (2002) and the fact that

$$
\sum_{t=1}^{T}\left(u_{t-i} u_{t-j}-\gamma_{i-j}\right)=\sum_{t=1}^{T}\left(\varepsilon_{t-i} \varepsilon_{t-j}-\gamma_{i-j}\right)+\sum_{t=1}^{T}\left(\varepsilon_{t-i} r_{t-j}+r_{t-i} \varepsilon_{t-j}+r_{t-i} r_{t-j}\right)=O_{p}\left(T^{1 / 2}\right) .
$$

Part (c). Note that $\left\|\sum_{t=1}^{T} Z_{t, k} v_{t, k}\right\| \leq\left\|\sum_{t=1}^{T} Z_{t, k}\left(v_{t, k}-v_{t}^{*}\right)\right\|+\left\|\sum_{t=1}^{T} Z_{t, k} v_{t}^{*}\right\|$. For $q=1, \ldots, k$,

$$
\begin{aligned}
E\left|\sum_{t=1}^{T} u_{t-q}\left(v_{t, k}-v_{t}^{*}\right)\right|^{2} & =\sum_{t=1}^{T} \sum_{r=1}^{T} E\left|u_{t-q}\left(v_{t, k}-v_{t}^{*}\right) u_{r-q}\left(u_{k, r}^{*}-v_{r}^{*}\right)\right| \\
& =\sum_{t=1}^{T} \sum_{r=1}^{T} E\left|\left(\sum_{i=0}^{\infty} c_{i} v_{t-q-i}^{*}\right)\left(\sum_{j=k+1}^{\infty} c_{k, j} v_{t-j}^{*}\right)\left(\sum_{m=0}^{\infty} c_{m} v_{r-q-m}^{*}\right)\left(\sum_{n=k+1}^{\infty} c_{k, n} v_{r-n}^{*}\right)\right| \\
& \leq \sum_{t=1}^{T} \sum_{r=1}^{T}\left[\sum_{i=0}^{\infty} \sum_{j=k+1}^{\infty} \sum_{m=0}^{\infty} \sum_{n=k+1}^{\infty}\left|c_{i} c_{k, j} c_{m} c_{k, n}\right| E\left|v_{t-q-i}^{*} v_{t-j}^{*} v_{r-q-m}^{*} v_{r-n}^{*}\right|\right] \\
& \leq \sup _{t} E\left|v_{t}^{*}\right|^{4} \sum_{t=1}^{T} \sum_{r=1}^{T}\left[\sum_{i=0}^{\infty}\left|c_{i}\right| \sum_{j=k+1}^{\infty}\left|c_{k, j}\right| \sum_{m=0}^{\infty}\left|c_{m}\right| \sum_{n=k+1}^{\infty}\left|c_{k, n}\right|\right] \\
& =T^{2}\left(\sum_{i=0}^{\infty}\left|c_{i}\right|\right)^{2}\left(\sum_{j=k+1}^{\infty}\left|c_{k, j}\right|\right)^{2} \sup E\left|v_{t}^{*}\right|^{4}=O\left(T^{2} k^{-2 s}\right),
\end{aligned}
$$

as $v_{t}$ and $r_{t}^{*}$ have bounded fourth moments and $\sum_{j=k+1}^{\infty}\left|c_{k, j}\right|=o_{p}\left(k^{-s}\right)$. Therefore,

$$
\begin{aligned}
E\left\|\sum_{t=1}^{T} Z_{t, k}\left(v_{t, k}-v_{t}^{*}\right)\right\|^{2} & =E\left(\sum_{t=1}^{T} u_{t-1}\left(v_{t, k}-v_{t}^{*}\right)\right)^{2}+\cdots+E\left(\sum_{t=1}^{T} u_{t-k}\left(v_{t, k}-v_{t}^{*}\right)\right)^{2} \\
& =O\left(T^{2} k^{1-2 s}\right),
\end{aligned}
$$

and $\left\|\sum_{t=1}^{T} Z_{t, k}\left(v_{t, k}-v_{t}^{*}\right)\right\|=O_{p}\left(T k^{1 / 2-s}\right)$. Because $T k^{1 / 2-s}=o\left(T k^{-1 / 2}\right)$ due to Assumption $\mathcal{K}$, $\left\|\sum_{t=1}^{T} Z_{t, k}\left(v_{t, k}-v_{t}^{*}\right)\right\|=O_{p}\left(T k^{-1 / 2}\right)$.

Furthermore, we have $\left\|\sum_{t=1}^{T} Z_{t, k} v_{t}^{*}\right\|=\sqrt{\left(\sum_{t=1}^{T} u_{t-1} v_{t}^{*}\right)^{2}+\ldots+\left(\sum_{t=1}^{T} u_{t-k} v_{t}^{*}\right)^{2}}$. It can be shown that

$$
\sum_{t=1}^{T} u_{t-q} v_{t}^{*}=\sum_{t=1}^{T} \varepsilon_{t-q} v_{t}+\sum_{t=1}^{T} \varepsilon_{t-q} r_{t}^{*}+\sum_{t=1}^{T} r_{t-q} r_{t}^{*}+\sum_{t=1}^{T} r_{t-q} v_{t}=O_{p}\left(T^{1 / 2}\right), \text { for } q=1, \ldots, k
$$

Since $E\left(\sum_{t=1}^{T} \varepsilon_{t-q} v_{t}\right)^{2}=\sum_{t=1}^{T} E\left(\varepsilon_{t-q}^{2} v_{t}^{2}\right) \leq \sup \left\{E\left(\varepsilon_{t}^{2}\right)\right\} \sum_{t=1}^{T} E\left(v_{t}^{2}\right)=T \sup \left\{E\left(\varepsilon_{t}^{2}\right)\right\} \sigma^{2}=O(T)$, we have $\sum_{t=1}^{T} \varepsilon_{t-q} v_{t}=O_{p}\left(T^{1 / 2}\right)$. Similarly, $\sum_{t=1}^{T} r_{t-q} v_{t}=O_{p}\left(T^{1 / 2}\right)$ because $E\left(\sum_{t=1}^{T} r_{t-q} v_{t}\right)^{2}=$ $\sum_{t=1}^{T} E\left(r_{t-q}^{2} v_{t}^{2}\right) \leq \sup \left\{E\left(r_{t}^{2}\right)\right\} \sum_{t=1}^{T} E\left(v_{t}^{2}\right)=T \sup \left\{E\left(r_{t}^{2}\right)\right\} \sigma^{2}=O(T)$. Since $\sum_{t=1}^{T} \varepsilon_{t-q} r_{t}^{*}$ and $\sum_{t=1}^{T} r_{t-q} r_{t}^{*}$ are also $O_{p}\left(T^{1 / 2}\right)$, we have that $\sum_{t=1}^{T} u_{t-q} v_{t}^{*}=O_{p}\left(T^{1 / 2}\right)$ and $\left\|\sum_{t=1}^{T} Z_{t, k} v_{t}^{*}\right\|=$ 
$O_{p}\left(k^{1 / 2} T^{1 / 2}\right)$. Because $k^{1 / 2} T^{1 / 2}$ is $O\left(T k^{-1 / 2}\right)$ under Assumption $\mathcal{K},\left\|\sum_{t=1}^{T} Z_{t, k} v_{t}^{*}\right\|=O_{p}\left(T k^{-1 / 2}\right)$ and the proof is complete.

\section{A.3 Proof of Theorem 2 and related results}

Proof of Lemma 1. It follows from the consistency property of $s_{A R}^{2}(k)$, which can be established using the consistency of $\hat{\sigma}$ and $\hat{\alpha}(1)$, see the proof of Theorem 1.

Proof of Theorem 2. Part (i). The proof of Theorem 2(i) consists of two steps. First, we show how to construct a càdlàg process $\tilde{X}_{n}^{*}$ such that $\tilde{X}_{n}^{*} \stackrel{w}{\rightarrow} B_{c}^{\bar{c}}$ in probability. Second, we show that $\tilde{X}_{n}^{*}$ and $X_{n}^{*}$ are 'close', in the sense that $\sup _{s \in[0,1]}\left|\tilde{X}_{n}^{*}(s)-X_{n}^{*}(s)\right| \stackrel{p}{\rightarrow} 0$. Taken together, these two results imply that $X_{n}^{*} \stackrel{w}{\rightarrow} B_{c}^{\bar{c}}$ in probability, as required.

For the first part, in order to define $\tilde{X}_{n}^{*}$ it suffices to consider the following construction, for $t=1, \ldots, n$ :

$$
\tilde{X}_{t}^{*}:=\left\{\begin{array}{cc}
\bar{c} & \text { if } \tilde{X}_{t-1}^{*}+n^{-1 / 2} \varepsilon_{t}^{*}>\bar{c} \\
\underline{c} & \text { if } \tilde{X}_{t-1}^{*}+n^{-1 / 2} \varepsilon_{t}^{*}<\underline{c} \\
\tilde{X}_{t-1}^{*}+n^{-1 / 2} \varepsilon_{t}^{*} & \text { otherwise }
\end{array}\right.
$$

with initial condition $X_{0}=0$ and $\varepsilon_{t}^{*}$ as in (4.11). By setting $\tilde{X}_{n}^{*}(s):=\tilde{X}_{\lfloor n s\rfloor}^{*}$, since $n \rightarrow \infty$ we can proceed as in the proof of Theorem 6 of Cavaliere (2005) to obtain that $\tilde{X}_{n}^{*} \stackrel{w}{\rightarrow} B_{\underline{c}}^{\bar{c}}$. This completes the first part.

To show that $\sup _{s \in[0,1]}\left|\tilde{X}_{n}^{*}(s)-X_{n}^{*}(s)\right|=\max _{t=0,1, . ., n}\left|\tilde{X}_{t}^{*}-X_{t}^{*}\right|=o_{p}(1)$ we can make use of an inductive argument to prove that for all $t=0, \ldots, n,\left|\tilde{X}_{t}^{*}-X_{t}^{*}\right| \leq|\underline{\widehat{c}}-\underline{c}|+|\widehat{\bar{c}}-\bar{c}|$. By Lemma 1 and the normality assumption on $\varepsilon_{t}^{*}$, this implies that $\sup _{s \in[0,1]}\left|\tilde{X}_{n}^{*}(s)-X_{n}^{*}(s)\right| \leq|\underline{\hat{c}}-\underline{c}|+|\widehat{\bar{c}}-\bar{c}|$, as required. We consider the one-bound case only, i.e. we set $\bar{c}, \widehat{\bar{c}}=\infty$; the proof for the twobound case is substantially identical. Furthermore, we let (without loss of generality) $\underline{\hat{c}}>\underline{c}$. For $t=0$, the relation is trivially satisfied. Now, suppose that the relation holds at time $t-1$, i.e. $\left|\tilde{X}_{t-1}^{*}-X_{t-1}^{*}\right| \leq|\underline{\widehat{c}}-\underline{c}|$. To prove that the relation holds at time $t$ as well it is useful to distinguish the following cases.

(a) $X_{t-1}^{*}+n^{-1 / 2} \varepsilon_{t}^{*} \geq \underline{\widehat{c}}$ and $\tilde{X}_{t-1}^{*}+n^{-1 / 2} \varepsilon_{t}^{*} \geq \underline{c}$. In this case we have that

$$
\left|X_{t}^{*}-\tilde{X}_{t}^{*}\right|=\left|X_{t-1}^{*}+n^{-1 / 2} \varepsilon_{t}^{*}-\left(\tilde{X}_{t-1}^{*}+n^{-1 / 2} \varepsilon_{t}^{*}\right)\right|=\left|X_{t-1}^{*}-\tilde{X}_{t-1}^{*}\right| \leq|\underline{\widehat{c}}-\underline{c}|,
$$

as required.

(b) $X_{t-1}^{*}+n^{-1 / 2} \varepsilon_{t}^{*} \geq \underline{\widehat{c}}$ and $\tilde{X}_{t-1}^{*}+n^{-1 / 2} \varepsilon_{t}^{*}<\underline{c}$. This implies $\varepsilon_{t}^{*}<0, X_{t-1}^{*} \geq \tilde{X}_{t-1}^{*}$, and $X_{t}^{*} \geq \tilde{X}_{t}^{*}$. 
Therefore,

$$
\begin{aligned}
X_{t}^{*}-\tilde{X}_{t}^{*} & =X_{t-1}^{*}+n^{-1 / 2} \varepsilon_{t}^{*}-\underline{c}=X_{t-1}^{*}-\tilde{X}_{t-1}^{*}+\left(\tilde{X}_{t-1}^{*}+n^{-1 / 2} \varepsilon_{t}^{*}-\underline{c}\right) \\
& \leq X_{t-1}^{*}-\tilde{X}_{t-1}^{*} \leq \underline{\widehat{c}}-\underline{c} .
\end{aligned}
$$

(c) $X_{t-1}^{*}+n^{-1 / 2} \varepsilon_{t}^{*}<\underline{\widehat{c}}$ and $\tilde{X}_{t-1}^{*}+n^{-1 / 2} \varepsilon_{t}^{*} \geq \underline{c}$. Both $X_{t}^{*} \geq \tilde{X}_{t}^{*}$ and $X_{t}^{*}<\tilde{X}_{t}^{*}$ are possible. In the former case, $X_{t}^{*}-\tilde{X}_{t}^{*}=\underline{\widehat{c}}-\tilde{X}_{t}^{*} \leq \underline{\widehat{c}}-\underline{c}$. The latter case implies $X_{t-1}^{*}<\tilde{X}_{t-1}^{*}$, and thus,

$$
\begin{aligned}
\tilde{X}_{t}^{*}-X_{t}^{*} & =\left(\tilde{X}_{t-1}^{*}+n^{-1 / 2} \varepsilon_{t}^{*}\right)-\widehat{\widehat{c}}=\tilde{X}_{t-1}^{*}-X_{t-1}^{*}+X_{t-1}^{*}+n^{-1 / 2} \varepsilon_{t}^{*}-\widehat{\widehat{c}} \\
& \leq \underline{\widehat{c}}-\underline{c}+X_{t-1}^{*}+n^{-1 / 2} \varepsilon_{t}^{*}-\underline{\widehat{c}}=X_{t-1}^{*}+n^{-1 / 2} \varepsilon_{t}^{*}-\underline{c} \leq \underline{\widehat{c}}-\underline{c} .
\end{aligned}
$$

(d) $X_{t-1}^{*}+n^{-1 / 2} \varepsilon_{t}^{*}<\underline{\widehat{c}}$ and $\tilde{X}_{t-1}^{*}+n^{-1 / 2} \varepsilon_{t}^{*}<\underline{c}$. Then $X_{t}^{*}=\underline{\widehat{c}}$ and $\tilde{X}_{t}^{*}=\underline{c}$, which obviously implies that $\left|X_{t}^{*}-\tilde{X}_{t}^{*}\right| \leq|\underline{\widehat{c}}-\underline{c}|$.

Taken together (a)-(d) implies that $\left|X_{t}^{*}-\tilde{X}_{t}^{*}\right| \leq|\underline{\widehat{c}}-\underline{c}|$ at time $t$, hence completing the proof.

Part (ii). It follows from Part (i) using standard continuous mapping arguments.

\begin{tabular}{|c|c|c|c|c|c|c|c|c|c|c|c|c|c|c|c|c|}
\hline \multirow[t]{2}{*}{$c$} & \multirow[t]{2}{*}{$T$} & \multirow[t]{2}{*}{$\mathcal{A D F}_{\alpha}$} & \multicolumn{2}{|c|}{$\mathcal{A D \mathcal { F } _ { \alpha } ^ { * }}$} & \multirow[t]{2}{*}{$\mathcal{A D F}_{t}$} & \multicolumn{2}{|c|}{$\mathcal{A D \mathcal { F } _ { t } ^ { * }}$} & \multirow[t]{2}{*}{$\mathcal{M Z _ { \alpha }}$} & \multicolumn{2}{|c|}{$\mathcal{M} \mathcal{Z}_{\alpha}^{*}$} & \multirow[t]{2}{*}{$\mathcal{M \mathcal { Z } _ { t }}$} & \multicolumn{2}{|c|}{$\mathcal{M Z _ { t } ^ { * }}$} & \multirow[t]{2}{*}{$\mathcal{M S B}$} & \multicolumn{2}{|c|}{$\mathcal{M S B}^{*}$} \\
\hline & & & (a) & (b) & & (a) & (b) & & (a) & (b) & & (a) & (b) & & (a) & (b) \\
\hline & & \multicolumn{15}{|c|}{ Two symmetric bounds } \\
\hline \multirow[t]{2}{*}{$\infty$} & 100 & 4.2 & 4.2 & 5.3 & 5.3 & 5.3 & 5.0 & 3.0 & 3.0 & 5.3 & 3.0 & 3.0 & 4.9 & 3.0 & 3.0 & 5.2 \\
\hline & 500 & 5.0 & 5.0 & 5.2 & 5.6 & 5.6 & 5.3 & 4.8 & 4.8 & 5.2 & 5.0 & 5.0 & 5.4 & 4.5 & 4.5 & 5.2 \\
\hline \multirow[t]{2}{*}{0.8} & 100 & 9.3 & 4.4 & 6.2 & 11.2 & 5.8 & 5.6 & 7.0 & 2.8 & 6.2 & 7.1 & 3.2 & 5.6 & 4.5 & 2.4 & 5.8 \\
\hline & 500 & 11.6 & 4.9 & 5.5 & 11.3 & 5.2 & 5.4 & 11.1 & 4.5 & 5.5 & 10.4 & 4.7 & 5.4 & 7.8 & 4.1 & 5.4 \\
\hline \multirow[t]{2}{*}{0.6} & 100 & 15.7 & 4.3 & 6.5 & 14.5 & 5.9 & 6.1 & 12.5 & 2.6 & 6.5 & 9.2 & 2.7 & 5.9 & 11.4 & 2.4 & 7.0 \\
\hline & 500 & 18.4 & 4.4 & 5.3 & 14.6 & 4.8 & 5.3 & 17.8 & 4.0 & 5.3 & 13.4 & 4.0 & 5.3 & 17.2 & 4.1 & 5.5 \\
\hline \multirow[t]{2}{*}{0.4} & 100 & 26.1 & 3.4 & 5.9 & 20.5 & 5.4 & 5.6 & 21.4 & 1.4 & 5.9 & 12.7 & 1.6 & 5.5 & 25.0 & 1.5 & 6.5 \\
\hline & 500 & 30.2 & 4.3 & 5.4 & 20.4 & 4.7 & 5.3 & 29.1 & 3.8 & 5.4 & 18.9 & 3.8 & 5.3 & 33.8 & 3.6 & 5.5 \\
\hline & & \multicolumn{15}{|c|}{ One single bound } \\
\hline \multirow[t]{2}{*}{0.8} & 100 & 7.0 & 4.3 & 5.8 & 8.3 & 5.5 & 5.5 & 5.1 & 3.0 & 5.8 & 5.0 & 3.2 & 5.3 & 3.7 & 2.6 & 5.5 \\
\hline & 500 & 8.2 & 4.9 & 5.4 & 8.3 & 5.3 & 5.4 & 7.8 & 4.5 & 5.4 & 7.6 & 4.8 & 5.4 & 6.1 & 4.2 & 5.4 \\
\hline \multirow[t]{2}{*}{0.6} & 100 & 10.1 & 4.3 & 6.1 & 9.9 & 5.7 & 5.6 & 7.9 & 2.8 & 6.1 & 6.3 & 2.9 & 5.5 & 7.4 & 2.5 & 6.4 \\
\hline & 500 & 11.3 & 4.6 & 5.5 & 9.8 & 5.1 & 5.3 & 10.9 & 4.2 & 5.5 & 8.9 & 4.3 & 5.3 & 10.4 & 4.1 & 5.4 \\
\hline \multirow[t]{2}{*}{0.4} & 100 & 13.2 & 3.9 & 5.7 & 11.7 & 5.3 & 5.4 & 10.9 & 2.4 & 5.7 & 7.6 & 2.4 & 5.2 & 12.3 & 2.4 & 6.2 \\
\hline & 500 & 15.0 & 4.1 & 5.1 & 12.0 & 4.5 & 5.1 & 14.6 & 3.8 & 5.1 & 11.1 & 3.9 & 5.1 & 16.1 & 3.8 & 5.1 \\
\hline \multirow[t]{2}{*}{0.2} & 100 & 15.8 & 2.9 & 5.3 & 14.1 & 4.5 & 5.4 & 13.5 & 1.5 & 5.3 & 9.3 & 1.6 & 5.3 & 14.9 & 1.7 & 5.5 \\
\hline & 500 & 17.7 & 3.8 & 4.8 & 14.1 & 4.2 & 4.9 & 17.2 & 3.5 & 4.8 & 13.2 & 3.5 & 4.9 & 18.8 & 3.6 & 4.8 \\
\hline \multirow[t]{2}{*}{0} & 100 & 17.4 & 2.5 & 5.1 & 18.8 & 4.5 & 5.0 & 14.9 & 1.0 & 5.1 & 13.4 & 1.1 & 5.1 & 14.2 & 1.1 & 5.1 \\
\hline & 500 & 20.0 & 3.7 & 5.1 & 19.1 & 4.2 & 5.1 & 19.4 & 3.4 & 5.1 & 18.0 & 3.3 & 5.1 & 18.5 & 3.3 & 5.0 \\
\hline
\end{tabular}

Part (iii). It suffices to follow the proof of Theorem 5 in Hansen (2000).

Table 1: Finite-sample null rejection probabilities white noise model, one-bound at $-c$ and two bounds at $-c$ and $c$ cases

Notes: (i) Nominal 5\% asymptotic level. (ii) Tests based on OLS de-meaned data. (iii) Columns (a) and (b) denote simulation-based tests with $n=20,000$ (a), $n=T$ (b). 


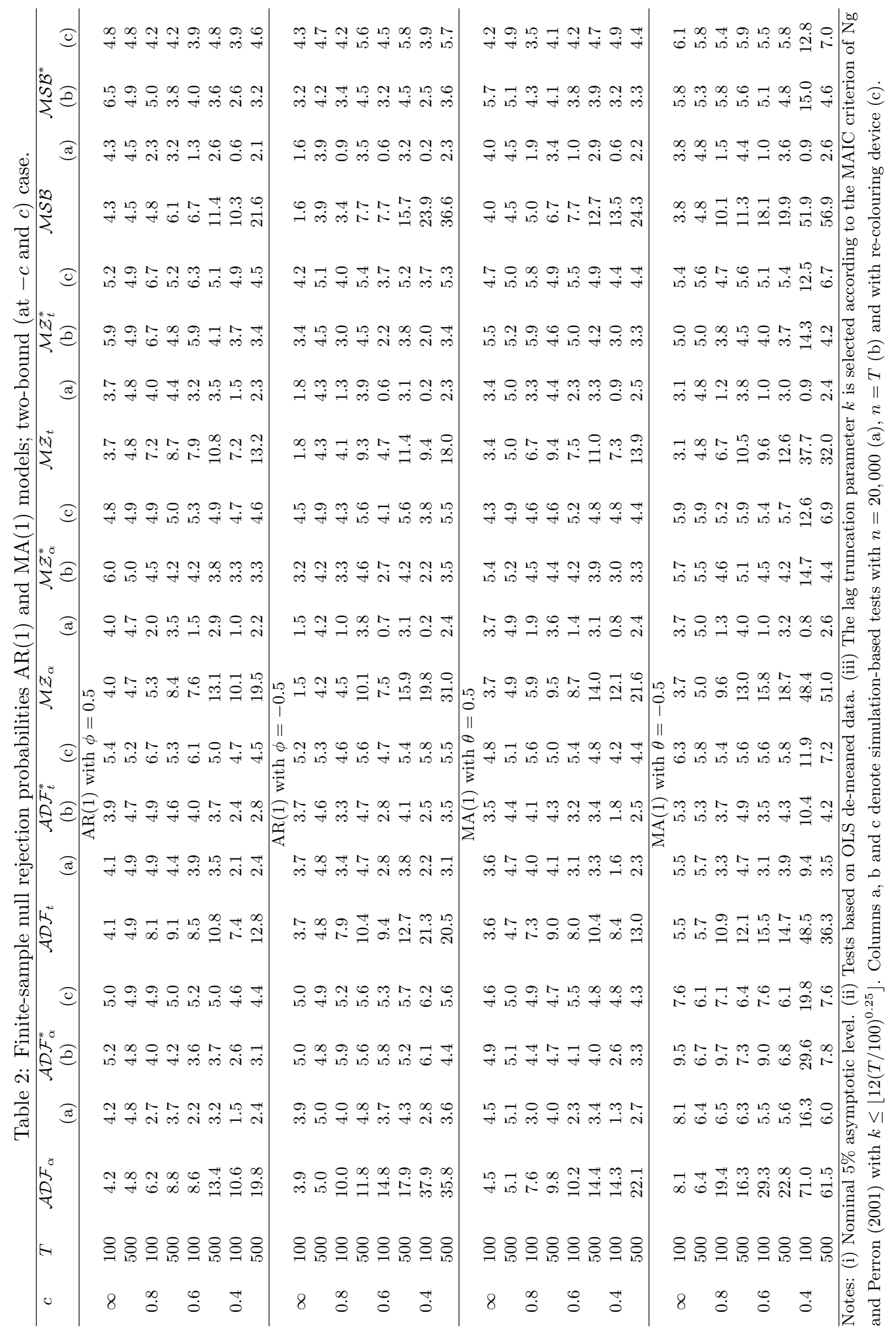




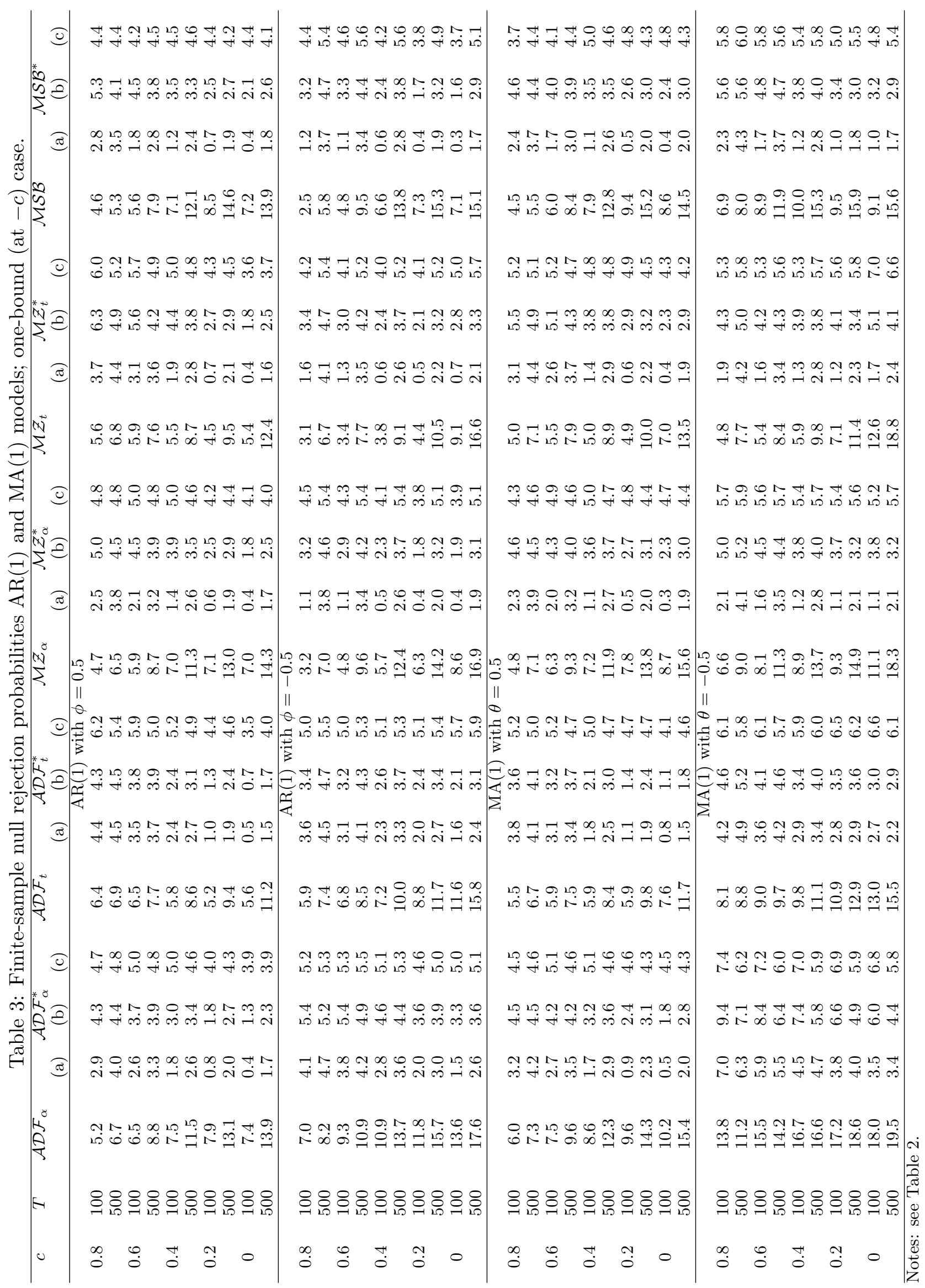


Table 4. Standard and simulation-based unit root tests, U.S. 3-month Treasury Bills rate, monthly data $1957-2008$

\begin{tabular}{|c|c|c|c|c|c|c|}
\hline \multicolumn{3}{|c|}{ Standard Unit Root Tests } & \multicolumn{4}{|c|}{ Simulation-based Unit Root Tests } \\
\hline & \multirow[t]{2}{*}{ statistic } & \multirow[t]{2}{*}{$p$-values } & & \multicolumn{3}{|c|}{$p$-values } \\
\hline & & & & (a) & (b) & (c) \\
\hline $\mathcal{A D F} \mathcal{F}_{\alpha}$ & -22.580 & 0.006 & $\mathcal{A D F}_{\alpha}^{*}$ & 0.073 & 0.068 & 0.086 \\
\hline $\mathcal{A D F}_{t}$ & -2.945 & 0.004 & $\mathcal{A D} \mathcal{F}_{t}^{*}$ & 0.142 & 0.128 & 0.118 \\
\hline $\mathcal{M \mathcal { Z } _ { \alpha }}$ & -25.195 & 0.003 & $\mathcal{M Z _ { \alpha } ^ { * }}$ & 0.053 & 0.061 & 0.088 \\
\hline $\mathcal{M \mathcal { Z } _ { t }}$ & -3.502 & 0.009 & $\mathcal{M Z _ { t } ^ { * }}$ & 0.057 & 0.061 & 0.092 \\
\hline $\mathcal{M S B}$ & 0.139 & 0.003 & $\mathcal{M S B}^{*}$ & 0.053 & 0.062 & 0.084 \\
\hline
\end{tabular}

Notes: (i) The number of lags determined by MAIC is $k=16$. Columns a, b and c denote simulation-based p-values with $n=20,000$ (a), $n=T$ (b) and with re-colouring device (c). (ii) The estimated long run variance is $s_{A R}^{2}(k)=0.63$. (iii) The estimated bound parameter is $\underline{\widehat{c}}=-0.16$.

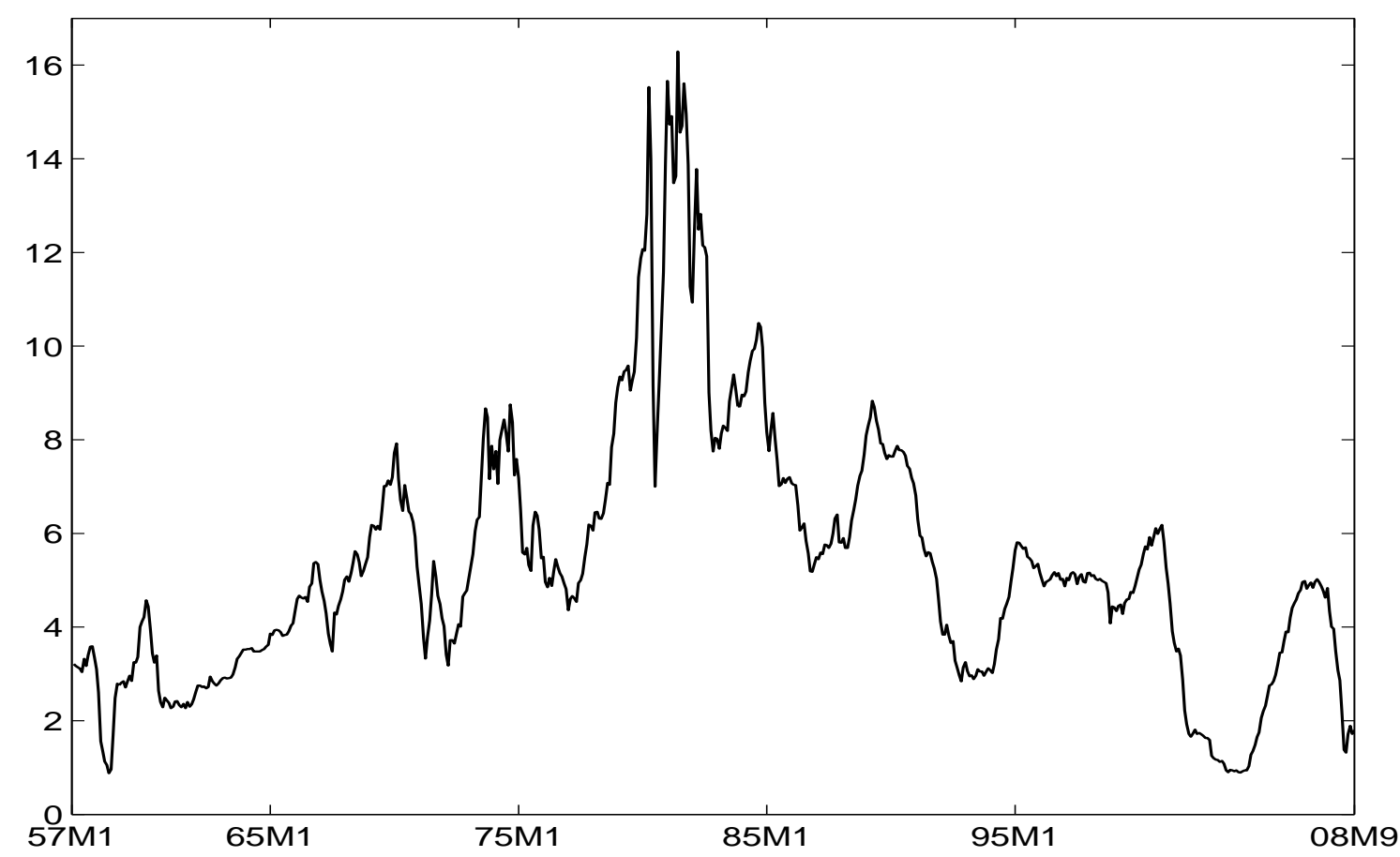

Figure 1: U.S. 3-month Treasury Bill rate, monthly data 1957:01-2008:09 